\title{
SOLITON DYNAMICS FOR FRACTIONAL SCHRÖDINGER EQUATIONS
}

\author{
SIMONE SECCHI AND MARCO SQUASSINA
}

\begin{abstract}
We investigate the soliton dynamics for the fractional nonlinear Schrödinger equation by a suitable modulational inequality. In the semiclassical limit, the solution concentrates along a trajectory determined by a Newtonian equation depending of the fractional diffusion parameter.
\end{abstract}

\section{INTRODUCTION}

In the last years, the study of fractional integrodifferential equations applied to physics as well as other areas has constantly grown. In $[16,21,22]$, the authors investigate recent developments in the description of anomalous diffusion via fractional dynamics and many fractional partial differential equations are derived asymptotically from Lévy random walk models, extending Brownian walk models in a natural way. In particular, in [19] a fractional Schrödinger equation was derived, extending to a Lévy framework a classical result that path integral over Brownian trajectories leads to the standard Schrödinger equation. We also refer the readers to [24] and to the references therein for further bibliography on the subject. Let $N \geq 1, s \in(0,1]$ and

$$
0<p<\frac{2 s}{N}
$$

Let $\mathrm{i}$ be the imaginary unit and let $V$ denote a smooth external time-independent potential. The goal of this paper is the study of the behaviour of the solution $u^{\varepsilon}: \mathbb{R}^{N} \rightarrow \mathbb{C}, \varepsilon>0$, to the Schrödinger equation involving the fractional laplacian $(-\Delta)^{s}$

$$
\left\{\begin{array}{l}
\mathrm{i} \varepsilon \frac{\partial u^{\varepsilon}}{\partial t}=\frac{\varepsilon^{2 s}}{2}(-\Delta)^{s} u^{\varepsilon}+V(x) u^{\varepsilon}-\left|u^{\varepsilon}\right|^{2 p} u^{\varepsilon} \quad \text { in }(0, \infty) \times \mathbb{R}^{N}, \\
u^{\varepsilon}(0, x)=Q\left(\frac{x-x_{0}}{\varepsilon}\right) e^{\frac{i}{\varepsilon}\left\langle x, v_{0}\right\rangle},
\end{array}\right.
$$

in the semi-classical limit $\varepsilon \rightarrow 0$, where $Q>0$ is the ground state of

$$
\frac{1}{2}(-\Delta)^{s} Q+Q=Q^{2 p+1}, \quad \text { in } \mathbb{R}^{N},
$$

and $x_{0}, v_{0} \in \mathbb{R}^{N}, v_{0} \neq 0$, are the initial position and velocity for the Newtonian type equation

$$
s|\dot{x}|^{2 s-2} \ddot{x}=-\nabla V(x), \quad x(0)=x_{0}, \quad \dot{x}(0)=v_{0} .
$$

In the limiting case $s=1$, rigorous results about the soliton dynamics of Schrödinger equation (1.1) were obtained in various papers, among which we mention the contributions by Bronski and Jerrard [3], Keraani [17] (see also [1,2,13] where a different technique is used) via arguments based upon the conservation laws satisfied by equation (1.1) and by the Newtonian ODE

$$
\ddot{x}=-\nabla V(x), \quad x(0)=x_{0}, \dot{x}(0)=v_{0},
$$

combined with the modulational stability estimates due to Weinstein [28,29]. Roughly speaking, the soliton dynamics occurs when, choosing an initial datum behaving like $Q\left(\left(x-x_{0}\right) / \varepsilon\right)$ the corresponding solution $u^{\varepsilon}(t)$ mantains the shape $Q((x-x(t)) / \varepsilon)$, up to an estimable error and locally in time, in the semi-classical transition $\varepsilon \rightarrow 0$. For a nice survey on solitons and

2000 Mathematics Subject Classification. 35Q51, 35Q40, 35Q41.

Key words and phrases. Soliton dynamics, fractional Schrödinger equation, ground states.

S. Secchi is supported by 2009 PRIN Critical Point Theory and Perturbative Methods for Nonlinear Differential Equations and by 2012 FIRB Dispersive equations and Fourier analysis, while M. Squassina is supported by 2009 PRIN Variational and Topological Methods in the Study of Nonlinear Phenomena. 
their stability features, see the work by Tao [25]. Concerning the well-posedness of problem (1.1) and a study of orbital stability of ground states, we refer the reader to $[14,15]$.

To the best of our knowledge, in the fractional case $s \in(0,1)$ neither modulational inequalities nor a soliton dynamics behavior have been investigated so far in the literature. Recently there have been many contributions concerning the properties of the solutions to problem (1.2), with a particular emphasis on the their qualitative behavior such as uniqueness, regularity, decays and - more important for our goals - the nondegeneracy, namely the linearized operator associated with (1.2) has an $N$-dimensional kernel which is spanned by $\left\{\partial Q / \partial x_{j}\right\}_{j=1, \ldots, N}$.

For these topics and the description of the physical background, we refer the reader to the works by Lenzmann and Frank [11] in the one-dimensional case, and the work by Lenzmann, Frank and Silvestre in the multi-dimentional setting [12]. See also the study of standing wave solutions in $[4,10]$, including symmetry and regularity features.

Let $\mathcal{E}: H^{s}\left(\mathbb{R}^{N}, \mathbb{C}\right) \rightarrow \mathbb{R}$ be the energy functional defined by

$$
\mathcal{E}(u):=\frac{1}{2} \int\left|(-\Delta)^{\frac{s}{2}} u\right|^{2}-\frac{1}{p+1} \int|u|^{2 p+2}
$$

and $\|\cdot\|_{H^{s}}$ denote the $H^{1}\left(\mathbb{R}^{N}, \mathbb{C}\right)$-norm. Then we have the following

Theorem 1.1. Assume that

$$
0<s<1, \quad 0<p<\frac{2 s}{N} .
$$

There exist positive constants $B, C$ independent of $\varepsilon \in(0,1]$ and $s \in(0,1)$ such that

$$
\mathcal{E}(\phi)-\mathcal{E}(Q) \geq C \inf _{x \in \mathbb{R}^{N}, \vartheta \in[0,2 \pi)}\left\|\phi-e^{\mathrm{i} \theta} Q(\cdot-x)\right\|_{H^{s}}^{2},
$$

for every $\phi \in H^{s}\left(\mathbb{R}^{N}, \mathbb{C}\right)$ such that $\mathcal{E}(\phi)-\mathcal{E}(Q) \leq B$.

This inequality is the fractional counterpart of an inequality which follows as a corollary of the results by M. Weinstein on Lyapunov stability for the nonlinear local Schrödinger equation, see $[28,29]$. A corresponding inequality for the nonlinear equations with a Hartree type nonlinearity was obtained in [6] based upon the nondegeneracy of ground states proved in [20].

Denoting $\|\cdot\|_{\mathcal{H}_{\varepsilon}^{s}}^{2}=\frac{1}{\varepsilon^{N-2 s}}\left\|(-\Delta)^{\frac{s}{2}} \cdot\right\|_{2}^{2}+\frac{1}{\varepsilon^{N}}\|\cdot\|_{2}^{2}$, we prove the following

Theorem 1.2. Let $u^{\varepsilon}(t) \in H^{s}\left(\mathbb{R}^{N} ; \mathbb{C}\right)$ denote the unique solution to the Cauchy problem (1.1). Then there exists a positive constant $C$, independent of $\varepsilon \in(0,1]$ and $s \in(0,1)$, such that

$$
\left\|(-\Delta)^{\frac{s}{2}} u^{\varepsilon}(t)\right\|_{2} \leq C \varepsilon^{\frac{N-2 s}{2}}
$$

for every $t \geq 0$ and every $\varepsilon>0$. Moreover, for any $\varepsilon>0$ sufficiently small and every $s \in(0,1)$ there exists a time $T^{\varepsilon, s}>0$ and continuous functions

$$
\theta^{\varepsilon, s}:\left[0, T^{\varepsilon, s}\right] \rightarrow \mathbb{R}, \quad z^{\varepsilon, s}: \mathbb{R}^{N} \rightarrow \mathbb{R}, \quad \mathscr{E}:\left[0, T^{\varepsilon, s}\right) \times(0,1] \times(0,1) \rightarrow \mathbb{R},
$$

such that, uniformly on $s \in(0,1]$,

$$
\mathscr{E}(0, \varepsilon, s)=\mathcal{O}\left(\varepsilon^{2}\right)
$$

and

$$
\left\|u^{\varepsilon}(t)-e^{\frac{i}{\varepsilon}\left(\langle x, v(t)\rangle+\theta^{\varepsilon, s}(t)\right)} Q\left(\frac{x-z^{\varepsilon, s}(t)}{\varepsilon}\right)\right\|_{\mathcal{H}_{\varepsilon}}^{2} \leq C \mathscr{E}(t, \varepsilon, s)+\mathcal{O}\left(\varepsilon^{2}\right) \quad \text { for all } t \in\left[0, T^{\varepsilon, s}\right) .
$$

Here $z^{\varepsilon, s}(t)=x(t)+\varepsilon \hat{z}^{\varepsilon, s}(t)$ for some continuous function $\hat{z}^{\varepsilon, s}: \mathbb{R}^{N} \rightarrow \mathbb{R}$, where $x(t)=x_{s}(t)$ is the solution to the Cauchy problem (1.3).

Hence, on a suitable time interval, the solution remains close to the initial profile with a term of order $\mathcal{O}\left(\varepsilon^{2}\right)$. It is expected that this qualitative behavior be preserved throughout the motion on finite time intervals and also that $z^{\varepsilon, s}(t)$ can be replaced by $x(t)$ (solving problem (1.3)) as in the local case. On the other hand, the proof of this claim seems out of reach because of the technical complications related to the nonlocal nature of $(-\Delta)^{s}$ (see also Remark 4.7).

Furthermore, we have the following 
Theorem 1.3. Let $u_{s}^{\varepsilon}(t) \in H^{s}\left(\mathbb{R}^{N} ; \mathbb{C}\right)$ denote the unique solution to the Cauchy problem $(1.1)$. Then it satisfies inequality (1.5). Let $T>0$ and assume that

$$
0<s<1, \quad 0<p<\frac{2 s}{N}
$$

that $V=V_{1}+V_{2}$ with $V_{1} \in \mathcal{C}^{3}\left(\mathbb{R}^{N}\right), V_{2} \in C^{4}\left(\mathbb{R}^{N}\right)$ and $V_{2}$ bounded from below. Then there exist a positive constant $C$ and a continuous function

$$
\mathscr{A}:[0, T] \times(0,1] \times(0,1) \rightarrow \mathbb{R},
$$

such that

$$
\lim _{s \rightarrow 1^{-}} \mathscr{A}(t, \varepsilon, s)=0, \quad \text { for all } t \in[0, T] \text { and } \varepsilon \in(0,1]
$$

and

$$
\left\|u_{s}^{\varepsilon}(t)-Q\left(\frac{x-x(t)}{\varepsilon}\right) e^{\mathrm{i} \frac{\langle v(t), x\rangle}{\varepsilon}}\right\|_{\mathcal{H}_{\varepsilon}^{s}}^{2} \leq C \varepsilon^{2 s}+\left\|u_{s}^{\varepsilon}(t)-u_{1}^{\varepsilon}(t)\right\|_{\mathcal{H}}^{2}+\mathscr{A}(t, \varepsilon, s), \quad \text { for all } t \in[0, T],
$$

where $x(t)=x_{s}(t)$ is the solution to (1.3), provided $x_{s}$ converges to $x_{1}$ on $[0, T]$.

Hence, on finite time intervals and precisely on the trajectory $x(t)$, the closeness estimate holds at the weaker rate $\varepsilon^{2 s}$ and in terms of the distance between the semigroups $u_{s}^{\varepsilon}$ and $u_{1}^{\varepsilon}$.

Remark 1.4. A major difficulty in our analysis is the lack of a point-wise calculus for fractional derivatives. In particular, the fractional laplacian does not obey a point-wise chain rule, nor a point-wise Leibniz rule for products. Only approximate versions of the fractional chain rule hold: see for instance [18, Lemma A10, Lemma A.11, Lemma A.12] and the references therein. This makes the analysis hard and we can prove the closedness of $u_{s}^{\varepsilon}$ to the orbit $Q((x-x(t) / \varepsilon)$ only when $s$ approaches the limit value $s=1$. We conjecture that the norm $\left\|u_{s}^{\varepsilon}(t)-u_{1}^{\varepsilon}(t)\right\|_{\mathcal{H}_{\varepsilon}^{s}}$ vanishes in the limit $s \rightarrow 1$, but the proof seems out of reach so far, as a regularity theory for the solutions to the fractional laplacian equation is still missing.

Remark 1.5. If $x(t)$ solves (1.3), then it is readily seen that the energy $t \mapsto \frac{1}{2}|\dot{x}(t)|^{2 s}+V(x(t))$ is a constant of motion. The Cauchy problems (1.3) and (1.4) are different from a dynamical viewpoint. For instance, (1.3) could fail to have uniqueness of solutions in the case $s \in(1 / 2,1]$ since $|\xi|^{2-2 s} \nabla V(x)$, where $\xi=\dot{x}$, could fail to be locally Lipschitz continuous. Also, it could admit heteroclinic connections, while (1.4) does not, as easy examples in the case $N=1$ show. To compare the behaviour of systems (1.3) and (1.4) in the physically relevant situation of harmonic potentials, let $N=2$ and $V\left(x_{1}, x_{2}\right):=\frac{1}{2} x_{1}^{2}+2 x_{2}^{2}$. Then $(1.3)$, for $s \in(0,1]$ is

$$
\left\{\begin{array}{l}
\dot{x}_{1}=\xi_{1}, \\
\dot{x}_{2}=\xi_{2}, \\
\dot{\xi}_{1}=-\frac{1}{s}\left(\xi_{1}^{2}+\xi_{2}^{2}\right)^{1-s} x_{1}, \\
\dot{\xi}_{2}=-\frac{4}{s}\left(\xi_{1}^{2}+\xi_{2}^{2}\right)^{1-s} x_{2},
\end{array}\right.
$$

with initial datum $x_{1}(0)=1, x_{2}(0)=a, \xi_{1}(0)=1$ and $\xi_{2}(0)=b$ for some $a, b>0$. See Figures 1-3 for the solutions to (1.6) for the cases $s=1,1 / 2,1 / 4$ respectively and data $a=1, b=1 / 2$ (left) and $a=1 / 2, b=1$ (right). Clearly, the complexity of the solutions increases as $s$ gets small. For any $s<1$, the system admits the stationary solutions of the form $(\alpha, \beta, 0,0)$ for $\alpha, \beta \in \mathbb{R}$, while for $s=1$ it only admits the trivial stationary solution $(0,0,0,0)$.

Remark 1.6. A numerical analysis of the soliton dynamics behaviour according to Theorem 1.2 is currently under investigation and it will be the subject of a forthcoming manuscript.

1.1. Fractional laplacian and notations. For the reader's convenience, we collect here some information about the fractional laplacian $(-\Delta)^{s}$ in $\mathbb{R}^{N}$. We define it as the pseudo-differential operator acting on $u \in \mathscr{S}\left(\mathbb{R}^{N}, \mathbb{C}\right)$ as

$$
(-\Delta)^{s} u:=\mathcal{F}^{-1}\left(|\xi|^{2 s} \mathcal{F} u(\xi)\right)
$$



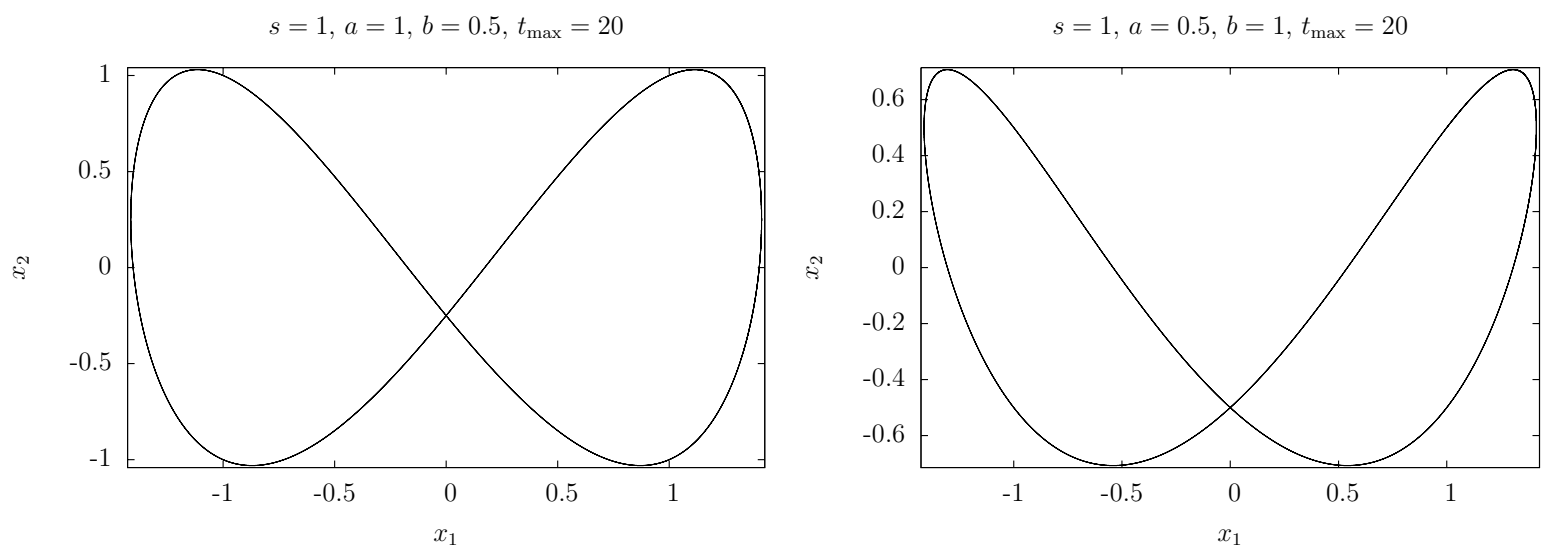

Figure 1. Solutions to (1.6) for $s=1$ with $a=1, b=0.5$ and $a=0.5, b=1$.
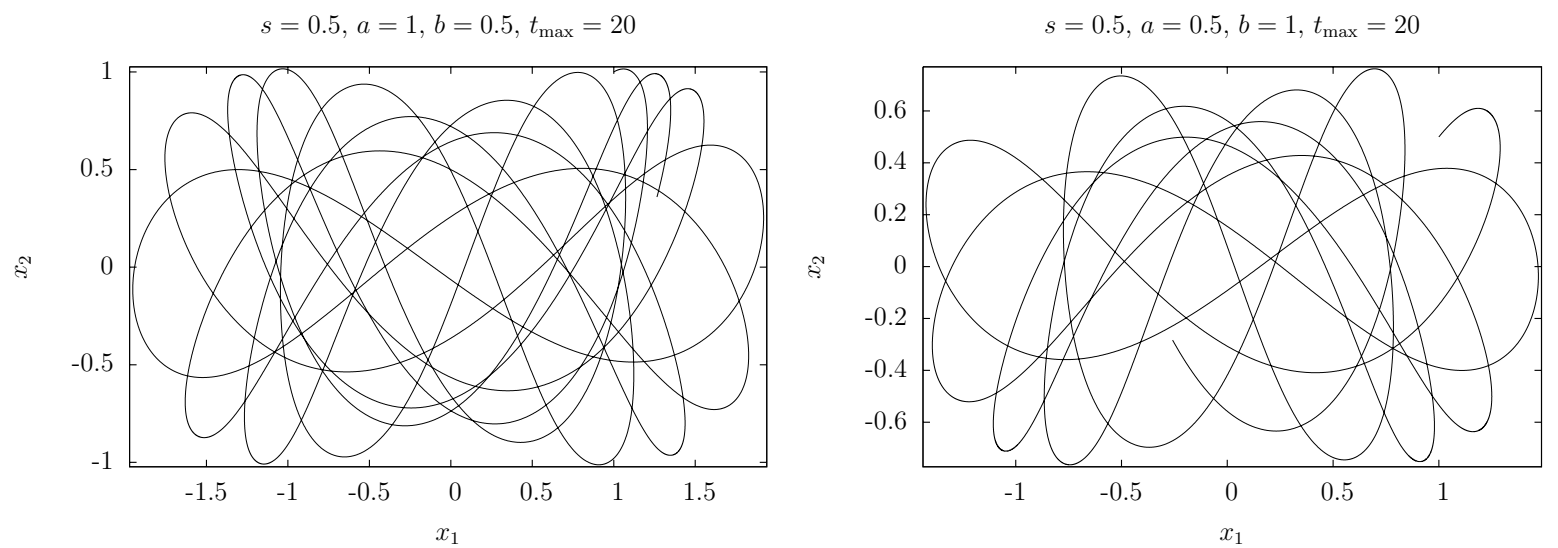

Figure 2. Solutions to (1.6) for $s=0.5$ with $a=1, b=0.5$ and $a=0.5, b=1$.
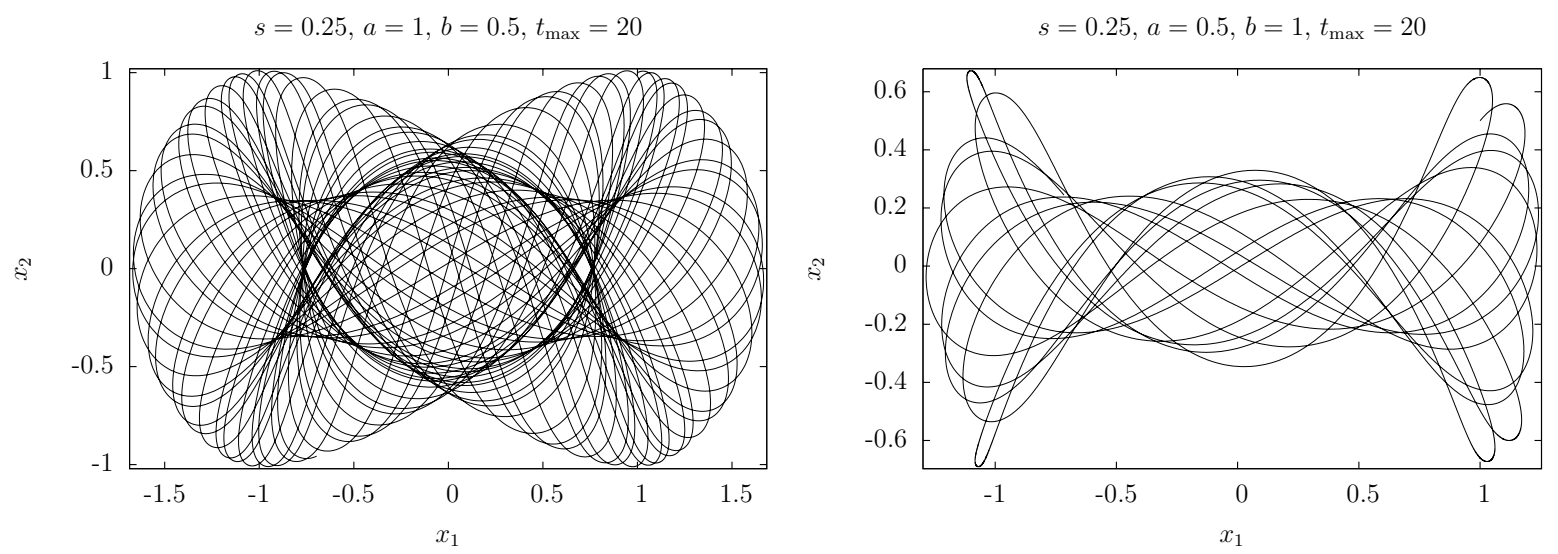

Figure 3. Solutions to (1.6) for $s=0.25$ with $a=1, b=0.5$ and $a=0.5, b=1$.

where $\mathcal{F}$ stands for the usual isometric Fourier transform in $L^{2}\left(\mathbb{R}^{N}, \mathbb{C}\right)$

$$
\mathcal{F}(u)(\xi)=\frac{1}{(2 \pi)^{N / 2}} \int e^{-i\langle x, \xi\rangle} u(x) d x
$$


As shown in [7, Section 3], equivalent definitions are

$$
\begin{aligned}
(-\Delta)^{s} u(x) & =C(N, s) P . V . \int \frac{u(x)-u(y)}{|x-y|^{N+2 s}} d y=C(N, s) \lim _{\varepsilon \rightarrow 0} \int_{\mathbb{R}^{N} \backslash B(0, \varepsilon)} \frac{u(x)-u(y)}{|x-y|^{N+2 s}} d y \\
& =-\frac{1}{2} C(N, s) \int \frac{u(x+y)+u(x-y)-2 u(x)}{|y|^{N+2 s}} d y
\end{aligned}
$$

where

$$
C(N, s)=\left(\int \frac{1-\cos \zeta_{1}}{|\zeta|^{N+2 s}} d \zeta\right)^{-1}
$$

Remark 1.7. In some papers, the fractional laplacian is defined without any reference to the constant $C(N, s)$. This is legitimate when $s$ is kept fixed, but we will see that the behavior of $C(N, s)$ as $s \rightarrow 1$ will play a crucial rôle in Section 4 .

The fractional Sobolev space $H^{s}\left(\mathbb{R}^{N}, \mathbb{C}\right)$ may be described as the set

$$
H^{s}\left(\mathbb{R}^{N}, \mathbb{C}\right)=\left\{\left.u \in L^{2}\left(\mathbb{R}^{N}, \mathbb{C}\right)\left|\int\left(1+\frac{1}{2}|\xi|^{2 s}\right)\right| \mathcal{F} u(\xi)\right|^{2} d \xi<+\infty\right\},
$$

endowed by the norm

$$
\|u\|_{H^{s}}^{2}=\|u\|_{2}^{2}+\frac{1}{2} \int|\xi|^{2 s}|\mathcal{F} u(\xi)|^{2} d \xi=\|u\|_{2}^{2}+\frac{1}{2}\left\|(-\Delta)^{\frac{s}{2}} u\right\|_{2}^{2} .
$$

An identical (squared) norm is

$$
\|u\|_{2}^{2}+\frac{C(N, s)}{4} \iint \frac{|u(x)-u(y)|^{2}}{|x-y|^{N+2 s}} d x d y
$$

and, see [7, Section 3],

$$
\lim _{s \rightarrow 0^{+}} \frac{C(N, s)}{s(1-s)}, \lim _{s \rightarrow 1^{-}} \frac{C(N, s)}{s(1-s)} \in(0,+\infty) .
$$

In the sequel, we will mainly work with the norm $\|u\|_{2}^{2}+\frac{1}{2}\left\|(-\Delta)^{\frac{s}{2}} u\right\|_{2}^{2}$. From the previous definitions, it follows that $\|\sqrt{-\Delta} u\|_{2}=\|\nabla u\|_{2}$ for any $u \in \mathscr{S}\left(\mathbb{R}^{N}\right)$.

Remark 1.8. By equations (2.8) and (2.9) in [7] and some elementary interpolation, we also deduce that the embeddings of $H^{s}\left(\mathbb{R}^{N}, \mathbb{C}\right)$ have constants that can be considered as independent of $s \in[\delta, 1], \delta>0$. This fact will be used several times in the sequel. Again from [7], we have that $(-\Delta)^{s} u$ converges pointwise to $-\Delta u$ as $s \rightarrow 1^{-}$, for all $u \in C_{c}^{\infty}\left(\mathbb{R}^{N}\right)$. Furthermore, for $u \in H^{1}\left(\mathbb{R}^{N}, \mathbb{C}\right)$,

$$
\lim _{s \rightarrow 1^{-}}\left\|(-\Delta)^{\frac{s}{2}} u\right\|_{2}=\|\nabla u\|_{2} .
$$

As a consequence, the fractional norms $\|u\|$ remain bounded as $s$ approaches 1 and the SobolevGagliardo-Nirenberg interpolation inequality

$$
\|u\|_{2 p+2} \leq C\|u\|_{2}^{\alpha}\left\|(-\Delta)^{\frac{s}{2}} u\right\|_{2}^{1-\alpha}, \quad \text { for all } u \in H^{s}\left(\mathbb{R}^{N}, \mathbb{C}\right),
$$

for a suitable $\alpha \in(0,1)$, holds with a contant $C$ which is independent of the choice of $s \in(\delta, 1]$.

\section{Notation}

(1) The usual euclidean scalar product of $\mathbb{R}^{N}$ will be denoted by $\langle x, y\rangle=\sum_{j=1}^{N} x_{j} y_{j}$.

(2) The space $\mathbb{C}$ will be endowed with the real inner product defined by

$$
z \cdot w=\mathfrak{R e}(z \bar{w})=\frac{z \bar{w}+\bar{z} w}{2}
$$

for every $z, w \in \mathbb{C}$.

(3) We will denote by $\|\cdot\|_{p}$ the $L^{p}$-norm in $\mathbb{R}^{N}$, and by $\|\cdot\|_{H^{s}}$ the $H^{s}$-norm in $\mathbb{R}^{N}$. These norms come from the inner products

$$
\langle u, v\rangle_{2}=\mathfrak{R e} \int u \bar{v} \quad \text { and } \quad\langle u, v\rangle_{H^{s}}=\frac{1}{2} \mathfrak{R e} \int(-\Delta)^{\frac{s}{2}} u \overline{(-\Delta)^{\frac{s}{2}} v}+\mathfrak{R e} \int u \bar{v}
$$

respectively. 
(4) Integrals over the whole space will be denoted by $\int$.

(5) Generic constants will be denoted by the letter $C$. We shall always assume that $C$ may vary from line to line but it is independent of $s$ and $\varepsilon$ unless explicitly stated.

(6) If $L$ is a linear operator acting on some space, the notation $\langle L, u\rangle$ denotes the value of $L$ evaluated at $u$. There is no confusion with the euclidean scalar product.

\section{Properties of Ground states}

A standing wave solution of the problem

is a function of the form

$$
\left\{\begin{array}{l}
\mathrm{i} \frac{\partial \phi}{\partial t}-\frac{1}{2}(-\Delta)^{s} \phi+|\phi|^{2 p} \phi=0, \\
\phi(0, x)=\phi_{0}(x)
\end{array}\right.
$$

$$
\phi(t, x)=e^{\mathrm{i} t} u(x),
$$

where $u: \mathbb{R}^{N} \rightarrow \mathbb{C}$ solves the elliptic equation

$$
\frac{1}{2}(-\Delta)^{s} u+u=|u|^{2 p} u \text {. }
$$

Definition 2.1. A solution $z: \mathbb{R}^{N} \rightarrow \mathbb{C}$ of (2.1) is called non-degenerate if the set of solutions $u$ of the linearized equation

$$
\frac{1}{2}(-\Delta)^{s} u+u=(2 p+1)|z|^{2 p} u
$$

is the $N$-dimensional subspace spanned by the partial derivatives of $z$.

We recall the following facts from $[9,12]$.

Theorem 2.2. Consider equation (2.1) for $0<s<1$ and $0<p<p_{\max }(s)$, where

$$
p_{\max }(s)= \begin{cases}\frac{2 s}{N-2 s} & \text { if } 0<s<N / 2 \\ +\infty & \text { otherwise. }\end{cases}
$$

Then the following facts hold.

(i) Existence. There exists a solution $Q \in H^{s}\left(\mathbb{R}^{N}\right)$ of equation (2.1) such that $Q$ is radially symmetric, positive and decreasing in $|x|$. Moreover, $Q$ is a ground state solution, namely a minimizer of the functional

$$
J^{s, p}(u)=\frac{\left(\int\left|(-\Delta)^{s / 2} u\right|^{2}\right)^{\frac{p}{2 s}}\left(\int|u|^{2}\right)^{\frac{p}{2 s}(2 s-1)+1}}{\int|u|^{2 p+2}} .
$$

(ii) Symmetry and monotonicity. If $Q \in H^{s}\left(\mathbb{R}^{N}\right)$ solves (2.1) with $Q \geq 0$ and $Q$ not identically equal to zero, then there exists $x_{0} \in \mathbb{R}^{N}$ such that $Q\left(\cdot-x_{0}\right)$ is radially simmetric, positive and decreasing in $\left|x-x_{0}\right|$.

(iii) Regularity and decay. If $Q \in H^{s}\left(\mathbb{R}^{N}\right)$ solves (2.1), then $Q \in H^{2 s+1}\left(\mathbb{R}^{N}\right)$. Moreover we have the decay estimate

$$
|Q(x)|+|x \cdot \nabla Q(x)| \leq \frac{C}{1+|x|^{N+2 s}}
$$

for all $x \in \mathbb{R}$ and some constant $C>0$.

(iv) Nondegeneracy. Suppose $Q \in H^{s}\left(\mathbb{R}^{N}\right)$ is a solution of (2.1), and consider the linearized operator at $Q$

$$
L_{+}=\frac{1}{2}(-\Delta)^{s}+1-(2 p+1) Q^{2 p}
$$

acting on $L^{2}\left(\mathbb{R}^{N}\right)$. If $Q=Q(|x|)>0$ is a ground state solution of (2.1), then

$$
\operatorname{ker} L_{+}=\operatorname{span}\left\{\frac{\partial Q}{\partial x_{1}}, \ldots, \frac{\partial Q}{\partial x_{N}}\right\} \text {. }
$$


(v) Uniqueness. The ground state for (2.1) is unique (up to translations).

(vi) Stability. For every $s_{0} \in(0,1]$ and $Q=Q_{s}$, we have

$$
\sup _{s \in\left(s_{0}, 1\right]}\left\|Q_{s}\right\|_{\infty}<\infty, \quad \sup _{s \in\left(s_{0}, 1\right]}\left\|Q_{s}\right\|_{2}<\infty, \quad \sup _{s \in\left(s_{0}, 1\right]}\left\|(-\Delta)^{s / 2} Q_{s}\right\|_{H^{s}}<\infty .
$$

Remark 2.3. In the sequel, we will often write $Q$ instead of $Q_{s}$, when $s$ is kept fixed.

Let us introduce some notation.

$$
\begin{aligned}
I(u) & =\frac{1}{2} \mathcal{E}(u)+\frac{1}{2}\|u\|_{2}^{2} \\
\mathcal{M}_{\gamma} & =\left\{u \in H^{s}\left(\mathbb{R}^{N}\right) \mid\|u\|_{2}^{2}=\gamma\right\} \\
K_{\mathcal{E}} & =\left\{c<0 \mid \mathcal{E}(u)=2 c, \nabla_{\mathcal{M}_{\gamma}} \mathcal{E}(u)=0 \text { for some } u \in \mathcal{M}_{\gamma}\right\} \\
\widetilde{K}_{\mathcal{E}} & =\left\{u \in \mathcal{M}_{\gamma} \mid \nabla_{\mathcal{M}_{\gamma}} \mathcal{E}(u)=0, \mathcal{E}(u)<0\right\} \\
K_{I} & =\left\{m \in \mathbb{R} \mid I(u)=m \text { and } I^{\prime}(u)=0 \text { for some } u \in \mathcal{N}\right\} \\
\widetilde{K}_{I} & =\left\{u \in \mathcal{N} \mid I^{\prime}(u)=0\right\},
\end{aligned}
$$

where

$$
\mathcal{N}=\left\{u \in H^{s}\left(\mathbb{R}^{N}\right) \mid\left\langle I^{\prime}(u), u\right\rangle=0\right\}
$$

is the Nehari manifold associated to (2.1). For future reference, we record that, for any $\xi \in$ $H^{s}\left(\mathbb{R}^{N}, \mathbb{C}\right)$ and any $\zeta \in H^{s}\left(\mathbb{R}^{N}, \mathbb{C}\right)$ there results

$$
\left\langle I^{\prime \prime}(\xi) \zeta, \zeta\right\rangle_{H^{s}}=\|\zeta\|_{H^{s}}^{2}-2 p \int\left(|\xi|^{2 p-2}(\xi \cdot \zeta) \xi\right) \cdot \zeta-\int|\xi|^{2 p} \zeta \cdot \zeta,
$$

where we have used the notation introduced in (1.8).

Definition 2.4. In the sequel, given a function $u$ and $\lambda, \mu \in \mathbb{R}$, we will write $u^{\mu, \lambda}(x)=\mu u(\lambda x)$.

Lemma 2.5. Given $u \in H^{s}\left(\mathbb{R}^{N}\right)$, the following scaling relations hold true:

$$
\begin{aligned}
\left\|u^{\mu, \lambda}\right\|_{2}^{2} & =\mu^{2} \lambda^{-N}\|u\|_{2}^{2}, \\
\left\|u^{\mu, \lambda}\right\|_{2 p+2}^{2 p+2} & =\mu^{2 p+2} \lambda^{-N}\|u\|_{2 p+2}^{2 p+2}, \\
\left\|(-\Delta)^{\frac{s}{2}} u^{\mu, \lambda}\right\|_{2}^{2} & =\mu^{2} \lambda^{2 s-N}\left\|(-\Delta)^{\frac{s}{2}} u\right\|_{2}^{2} .
\end{aligned}
$$

Proof. The three identities follow from a direct computation.

Lemma 2.6. Assume that

$$
0<s<1, \quad 0<p<\frac{2 s}{N} .
$$

Then there is a bijective correspondence between the sets $\widetilde{K}_{\mathcal{E}}$ and $\widetilde{K}_{I}$.

Proof. Let us pick $v \in \mathcal{M}_{\gamma}$ such that $\left\langle\mathcal{E}^{\prime}(v), v\right\rangle=-\ell \gamma$ and $\mathcal{E}(v)=2 c<0$. Then $-\ell \gamma-4 c=$ $\left\langle\mathcal{E}^{\prime}(v) v\right\rangle-2 \mathcal{E}(v)=-\frac{2 p}{p+1}\|u\|_{2 p+2}^{2 p+2}<0$, and therefore $\ell>0$. We can define a map $T^{\mu, \lambda}: \mathcal{M}_{\gamma} \rightarrow \mathcal{N}$ by $T^{\mu, \lambda}(v)=v^{\mu, \lambda}$, where $\mu$ and $\lambda$ are defined by the condition

$$
\lambda=\ell^{-\frac{1}{2 s}}, \quad \mu=\ell^{-\frac{1}{2 p}} .
$$

It is easy to check that $v^{\mu, \lambda} \in \widetilde{K}_{I}$. Viceversa, if $u \in \widetilde{K}_{I}$, then we choose $\ell>0$ such that

$$
\ell^{\frac{1}{p}-\frac{N}{2 s}}=\frac{\gamma}{\|u\|_{2}^{2}}, \quad \lambda=\ell^{\frac{1}{2 s}}, \quad \mu=\ell^{\frac{1}{2 p}}
$$

so that $u^{\mu, \lambda} \in \mathcal{M}_{\gamma}$ and $\nabla_{\mathcal{M}_{\gamma}} \mathcal{E}\left(u^{\mu, \lambda}\right)=0$. Whence $\left(T^{\mu, \lambda}\right)^{-1}=T^{1 / \mu, 1 / \lambda}$ concluding the proof. 
Lemma 2.7. Assume that

$$
0<s<1, \quad 0<p<\frac{2 s}{N} .
$$

Then there exists a bijective correspondence $\mathscr{T}: K_{I} \rightarrow K_{\mathcal{E}}$ defined by the formula

$$
\mathscr{T}(m)=\left(\frac{N}{2 s}-\frac{1}{p}\right)\left(\frac{\gamma s p}{2(p+1) s-N p}\right)^{1+\frac{2 s p}{2 s-N p}}\left(\frac{1}{m}\right)^{\frac{2 s p}{2 s-N p}} .
$$

Proof. Pick $m \in K_{I}$. Then, there is some $u \in \mathcal{N}$ such that $I(u)=m$ and $I^{\prime}(u)=0$. Therefore

$$
m=I(u)-\frac{1}{2 p+2}\left\langle I^{\prime}(u), u\right\rangle=\frac{1}{2}\left(1-\frac{1}{p+1}\right)\|u\|_{H^{s}}^{2}>0 .
$$

For $c \in K_{\mathcal{E}} \cap \mathbb{R}^{-}$we select $v \in \mathcal{M}_{\gamma}$ corresponding to $c$. In turn, there exists $\ell>0$ such that $\frac{1}{2}(-\Delta)^{s} v-|v|^{2 p} v=-\ell v$. Let us set $T^{\mu, \lambda}(v)=v^{\mu, \lambda}$ with $\lambda=\ell^{-1 /(2 s)}$ and $\mu=\ell^{-1 /(2 p)}$. Then, $T^{\mu, \lambda}$ maps $\mathcal{M}_{\gamma}$ into $\mathcal{N}$ and $v^{\mu, \lambda}$ solves $\frac{1}{2}(-\Delta)^{s} v^{\mu, \lambda}+v^{\mu, \lambda}=\left|v^{\mu, \lambda}\right|{ }^{2 p} v^{\mu, \lambda}$. The Pohǒzaev identity yields

$$
\frac{N-2 s}{4} \int\left|(-\Delta)^{\frac{s}{2}} v^{\mu, \lambda}\right|^{2}+\frac{N}{2}\left\|v^{\mu, \lambda}\right\|_{2}^{2}=\frac{N}{2 p+2}\left\|v^{\mu, \lambda}\right\|_{2 p+2}^{2 p+2}
$$

But $v^{\mu, \lambda} \in \mathcal{N}$, namely

$$
\left\|v^{\mu, \lambda}\right\|_{2}^{2}+\frac{1}{2} \int\left|(-\Delta)^{\frac{s}{2}} v^{\mu, \lambda}\right|^{2}=\int\left|v^{\mu, \lambda}\right|^{2 p+2}
$$

Hence

$$
\left(\frac{N-2 s}{4}-\frac{N}{4 p+4}\right)\left\|(-\Delta)^{\frac{s}{2}} v^{\mu, \lambda}\right\|_{2}^{2}+\left(\frac{N}{2}-\frac{N}{2 p+2}\right)\left\|v^{\mu, \lambda}\right\|_{2}^{2}=0
$$

and

$$
\left(\frac{1}{4}-\frac{1}{4 p+4}\right)\left\|(-\Delta)^{\frac{s}{2}} v^{\mu, \lambda}\right\|_{2}^{2}+\left(\frac{1}{2}-\frac{1}{2 p+2}\right)\left\|v^{\mu, \lambda}\right\|_{2}^{2}=m,
$$

where $m=I\left(v^{\mu, \lambda}\right)$. After trivial manipulations, we discover that

$$
\begin{aligned}
\left\|(-\Delta)^{\frac{s}{2}} v^{\mu, \lambda}\right\|_{2}^{2} & =\frac{2 N m}{s}, \\
\left\|v^{\mu, \lambda}\right\|_{2}^{2} & =\frac{2 m s(p+1)-N m p}{s p}, \\
\left\|v^{\mu, \lambda}\right\|_{2 p+2}^{2 p+2} & =\frac{2 m(p+1)}{p} .
\end{aligned}
$$

Recalling Lemma 2.5, we write the previous identities as

$$
\begin{aligned}
\frac{\mu^{2}}{\lambda^{N-2 s}}\left\|(-\Delta)^{\frac{s}{2}} v\right\|_{2}^{2} & =\frac{m N}{s}, \\
\frac{\mu^{2 p+2}}{\lambda^{N}} \frac{1}{2 p+2} \int|v|^{2 p+2} & =\frac{m}{p}, \\
\frac{\mu^{2}}{\lambda^{N}}\|v\|_{2}^{2} & =\frac{2 m(p+1) s-m N p}{s p} .
\end{aligned}
$$

But $v \in \mathcal{M}_{\gamma}$, and hence

$$
\gamma=\|v\|_{2}^{2}=\ell^{\frac{1}{p}-\frac{N}{2 s}} \frac{2 m(p+1) s-m N p}{s p},
$$

and

$$
\ell^{\frac{2 s-N p}{2 s p}}=\frac{\gamma s p}{2 m(p+1) s-m N p} .
$$

Since $\lambda=\ell^{-\frac{1}{2 s}}, \mu=\ell^{-\frac{1}{2 p}}$, we find

$$
\left\|(-\Delta)^{\frac{s}{2}} v\right\|_{2}^{2}=\frac{\lambda^{N-2 s}}{\mu^{2}} \frac{2 m N}{s}=\left(\frac{\gamma s p}{2(p+1) s-N p}\right)^{1+\frac{2 s p}{2 s-N p}} \frac{2 N}{s} m^{-\frac{2 s p}{2 s-N p}} .
$$


Similarly,

$$
\frac{1}{2 p+2}\|v\|_{2 p+2}^{2 p+2}=\frac{\lambda^{N}}{\mu^{2 p+2}} \frac{m}{p}=\frac{1}{p}\left(\frac{\gamma s p}{2(p+1) s-N p}\right)^{1+\frac{2 s p}{2 s-N p}}\left(\frac{1}{m}\right)^{\frac{2 s p}{2 s-N p}} .
$$

To summarize, if $c<0$ is a constrained critical value of $\mathcal{E}$ on $\mathcal{M}_{\gamma}$ and $m$ is the corresponding critical value of $I$, then $c$ is given by

$$
c=\left(\frac{N}{2 s}-\frac{1}{p}\right)\left(\frac{\gamma s p}{2(p+1) s-N p}\right)^{1+\frac{2 s p}{2 s-N p}}\left(\frac{1}{m}\right)^{\frac{2 s p}{2 s-N p}} .
$$

This concludes the proof.

We also have the following

Corollary 2.8. Assume that

$$
0<s<1, \quad 0<p<\frac{2 s}{N}, \quad \gamma_{0}:=m_{\mathcal{N}} \frac{2(p+1) s-N p}{s p}, \quad m_{\mathcal{N}}:=\inf _{u \in \mathcal{N}} I(u) .
$$

Then we have

$$
m_{\mathcal{N}}=\inf _{u \in \mathcal{M}_{\gamma_{0}}} I(u)=: m_{\gamma_{0}}
$$

Furthermore, any $u_{0} \in \mathcal{N}$ with $I\left(u_{0}\right)=m_{\mathcal{N}}$ satisfies $\left\|u_{0}\right\|_{2}^{2}=\gamma_{0}$ and $\mathcal{E}\left(u_{0}\right)=\inf _{u \in \mathcal{M}_{\gamma_{0}}} \mathcal{E}(u)$.

Proof. Observe that, taking into account the monotonocity of $\mathscr{T}$, we obtain

$$
\begin{aligned}
m_{\gamma_{0}} & =\inf _{u \in \mathcal{M}_{\gamma_{0}}} \frac{1}{2} \mathcal{E}(u)+\frac{\gamma_{0}}{2}=\mathscr{T}\left(m_{\mathcal{N}}\right)+\frac{\gamma_{0}}{2} \\
& =\left(\frac{N}{2 s}-\frac{1}{p}\right)\left(\frac{\gamma_{0} s p}{2(p+1) s-N p}\right)^{1+\frac{2 s p}{2 s-N p}}\left(\frac{1}{m_{\mathcal{N}}}\right)^{\frac{2 s p}{2 s-N p}}+\frac{\gamma_{0}}{2}=m_{\mathcal{N}},
\end{aligned}
$$

after a few computations and by the value of $\gamma_{0}$. This concludes the proof of the first assertion. Now, given $u_{0} \in \mathcal{N}$ with $I\left(u_{0}\right)=m_{\mathcal{N}}$, by repeating the argument in the proof of Lemma 2.7 (namely by combining the energy, the Pohozaev and the Nehari identities) and by the definition of $\gamma_{0}$ we get $\left\|u_{0}\right\|_{2}^{2}=\gamma_{0}$ (notice that, from (2.3), it holds $\ell=1=\lambda=\mu$, i.e. $T^{\mu, \lambda}=T^{1 / \mu, 1 / \lambda}=$ Id). The last assertion then follows immediately from $m_{\mathcal{N}}=m_{\gamma_{0}}$.

Corollary 2.9. Let $Q>0$ be the unique ground state solution to problem (1.2) and let $s, p$ and $\gamma_{0}$ be as in (2.4). Then we have

$$
\mathcal{E}(Q)=\min \left\{\mathcal{E}(q): q \in H^{s}\left(\mathbb{R}^{N}, \mathbb{C}\right),\|q\|_{2}=\gamma_{0}=\|Q\|_{2}\right\},
$$

and $\min \left\{\mathcal{E}(q): q \in H^{s}\left(\mathbb{R}^{N}, \mathbb{C}\right),\|q\|_{2}=\|Q\|_{2}\right\}$ admits a unique solution.

Proof. The assertion follows by Corollary 2.8 and by the uniqueness of ground state solutions.

\section{Spectral ANALYSis of Linearization}

In this section we perform a spectral analysis of the linearized operator at a non degenerate ground state $Q$

$$
L_{+}=\frac{1}{2}(-\Delta)^{s}+1-(2 p+1) Q^{2 p}
$$

acting on $L^{2}\left(\mathbb{R}^{N}, \mathbb{C}\right)$. Let us introduce the closed subspaces of $H^{s}\left(\mathbb{R}^{N}, \mathbb{C}\right)$

$$
\begin{aligned}
\mathcal{V} & =\left\{u \in H^{s}\left(\mathbb{R}^{N}, \mathbb{C}\right) \mid\langle u, Q\rangle_{2}=0\right\} \\
\mathcal{V}_{0} & =\left\{u \in H^{s}\left(\mathbb{R}^{N}, \mathbb{C}\right) \mid\langle u, Q\rangle_{2}=\left\langle u, H(Q) \frac{\partial Q}{\partial x_{j}}\right\rangle_{2}=0, j=1,2, \ldots, N\right\},
\end{aligned}
$$

where $H(Q)=(2 p+1) Q^{2 p}$. 
Lemma 3.1. Assume that

and define

$$
0<s<1, \quad 0<p<\frac{2 s}{N}
$$

Then $\alpha>0$.

$$
\alpha=\inf \left\{\left\langle L_{+}(u), u\right\rangle \mid u \in \mathcal{V}_{0},\|u\|_{2}=1\right\} .
$$

Proof. Firstly, we claim that $\alpha \geq 0$. Indeed, $\partial Q / \partial x_{j} \in \mathcal{V}$ for each $j=1, \ldots, N$, and

$$
\left\langle L_{+}\left(\partial Q / \partial x_{j}\right), \partial Q / \partial x_{j}\right\rangle=0 \text {. }
$$

In addition, since (see Corollary 2.8) $Q$ minimizes $\mathcal{E}(u)$ over the constraint $\mathcal{M}=\{u \in$ $\left.H^{s}\left(\mathbb{R}^{N}, \mathbb{C}\right) \mid\|u\|_{2}=\|Q\|_{2}\right\}$, it follows that $Q$ also minimizes $2 I(u)=\mathcal{E}(u)+\|u\|_{2}^{2}$ over the same constraint. In particular, $Q$ is a constrained critical point of $I$, and a direct computation shows that the second derivative $I^{\prime \prime}(Q)$ is positive semi-definite on $\mathcal{V}$. Therefore

$$
\inf \left\{\left\langle L_{+}(u), u\right\rangle \mid u \in \mathcal{V}\right\}=0 \text {. }
$$

Since

$$
\alpha \geq \inf \left\{\left\langle L_{+}(u), u\right\rangle \mid u \in \mathcal{V}\right\}
$$

the claim is proved. We assume now, for the sake of contradiction, that $\alpha=0$. Pick any minimizing sequence $\left\{u_{n}\right\}_{n}$ for $\alpha$, so that $\left\|u_{n}\right\|_{2}=1$ for every $n \in \mathbb{N}, u_{n} \in \mathcal{V}_{0}$ and $\left\langle L_{+}\left(u_{n}\right), u_{n}\right\rangle=o(1)$ as $n \rightarrow \infty$. On the other hand,

$$
\left\langle L_{+}\left(u_{n}\right), u_{n}\right\rangle=\frac{1}{2} \int\left|(-\Delta)^{\frac{s}{2}} u_{n}\right|^{2}+\int\left|u_{n}\right|^{2}-(2 p+1) \int Q^{2 p}\left|u_{n}\right|^{2},
$$

and hence

$$
\int_{\mathbb{R}^{N}}\left|(-\Delta)^{\frac{s}{2}} u_{n}\right|^{2} \leq C\left(o(1)+(2 p+1) \int Q^{2 p}\left|u_{n}\right|^{2}\right) \leq C+C \int\left|u_{n}\right|^{2} \leq C .
$$

The sequence $\left\{u_{n}\right\}_{n}$ being bounded in $H^{s}\left(\mathbb{R}^{N}, \mathbb{C}\right)$, we can assume without loss of generality that $u_{n} \rightarrow u$ in $H^{s}\left(\mathbb{R}^{N}, \mathbb{C}\right)$, and $u \in \mathcal{V}_{0}$ because $\mathcal{V}_{0}$ is weakly closed.

Notice that the operator $\{u \mapsto H(Q) u\}$ is a multiplication operator by the function $Q^{2 p}$ which tends to zero at infinity. Given $\rho>0$, let us write

$$
\chi_{\rho}(x)= \begin{cases}1 & \text { if }|x| \leq \rho \\ 0 & \text { if }|x|>\rho\end{cases}
$$

It follows that

$$
\int Q^{2 p}|u|^{2}-\left|\chi_{\rho} Q\right|^{2 p}|u|^{2}=\int_{\mathbb{R}^{N} \backslash B(0, \rho)} Q^{2 p}|u|^{2} \leq \sup _{x \in \mathbb{R}^{N} \backslash B(0, \rho)} Q(x)^{2 p} \int|u|^{2} .
$$

Then the compact embedding of $H^{s}(B(0, \rho))$ into $L^{2}(B(0, \rho))$ yields the compactness of the multiplication operator $H(Q)$ (see also [27, Theorem 10.20]) and the convergence $\left\langle u_{n}, H(Q) u_{n}\right\rangle_{2}=$ $\langle u, H(Q) u\rangle_{2}+o(1)$. As a consequence,

$$
0 \leq\left\langle L_{+}(u), u\right\rangle \leq \liminf _{n \rightarrow+\infty}\left(\left\|u_{n}\right\|_{H^{s}}^{2}-\left\langle u_{n}, H(Q) u_{n}\right\rangle_{2}\right)=\lim _{n \rightarrow+\infty}\left\langle L_{+}\left(u_{n}\right), u_{n}\right\rangle=0,
$$

forcing $\left\langle L_{+}(u), u\right\rangle=0$ and $\left\langle L_{+}\left(u_{n}\right), u_{n}\right\rangle=\left\langle L_{+}(u), u\right\rangle+o(1)$. By lower semicontinuity, we get

$$
\begin{aligned}
\|u\|_{H^{s}}^{2} & \leq \liminf _{n \rightarrow+\infty}\left\|u_{n}\right\|_{H^{s}}^{2} \leq \limsup _{n \rightarrow+\infty}\left\|u_{n}\right\|_{H^{s}}^{2}=\lim _{n \rightarrow+\infty}\left\langle L_{+}\left(u_{n}\right), u_{n}\right\rangle+\left\langle u_{n}, H(Q) u_{n}\right\rangle_{2} \\
& =\left\langle L_{+}(u), u\right\rangle+\langle u, H(Q) u\rangle_{2}=\|u\|_{H^{s}}^{2} .
\end{aligned}
$$

So far we have proved that $u_{n} \rightarrow u$ strongly in $H^{s}\left(\mathbb{R}^{N}, \mathbb{C}\right)$ and that $u$ is a minimizer for $\alpha$. From now on, for ease of notation, we assume that $N=1$; the general case is similar, but we need to replace $Q^{\prime}$ with either any partial derivative or with the gradient of $Q$ in the following arguments. Hence, the assumption reads as $p<2 s$. Let $\lambda, \mu$ and $\gamma$ be the Lagrange multipliers associated to $u$, so that, for all $v \in H^{s}\left(\mathbb{R}^{N}, \mathbb{C}\right)$,

$$
\left\langle L_{+} u, v\right\rangle=\lambda\langle u, v\rangle_{2}+\mu\langle Q, v\rangle_{2}+\gamma\left\langle H(Q) Q^{\prime}, v\right\rangle_{2} .
$$


Choosing $v=u \in \mathcal{V}_{0}$ immediately yields $\lambda=0$. Instead, choosing $v=Q^{\prime}$ and recalling also that $Q \perp Q^{\prime}$ in $L^{2}\left(\mathbb{R}^{N}, \mathbb{C}\right)$, we find

$$
0=\left\langle L_{+} u, Q^{\prime}\right\rangle=\mu\left\langle Q, Q^{\prime}\right\rangle_{2}+\gamma\left\langle H(Q) Q^{\prime}, Q^{\prime}\right\rangle_{2}=\gamma\left\langle H(Q) Q^{\prime}, Q^{\prime}\right\rangle_{2} .
$$

Now,

$$
\left\langle H(Q) Q^{\prime}, Q^{\prime}\right\rangle_{2}=(2 p+1) \int Q^{2 p}\left|Q^{\prime}\right|^{2}>0,
$$

and this yields $\gamma=0$. Hence $L_{+} u=\mu Q$. To proceed further, we compute

$$
L_{+}\left(x Q^{\prime}\right)=\frac{1}{2}(-\Delta)^{s}\left(x Q^{\prime}\right)+x Q^{\prime}-(2 p+1) Q^{2 p}\left(x Q^{\prime}\right)
$$

and we use the commutator identity (see [23, Remark 2.2] or [11, Lemma 5.1])

$$
(-\Delta)^{s}(x \cdot \nabla u)=2 s(-\Delta)^{s} u+x \cdot \nabla(-\Delta)^{s} u
$$

with $u=Q$, which implies

$$
(-\Delta)^{s}\left(x Q^{\prime}\right)-x(-\Delta)^{s} Q^{\prime}=2 s(-\Delta)^{s} Q .
$$

But $\frac{1}{2}(-\Delta)^{s} Q^{\prime}+Q^{\prime}-(2 p+1) Q^{2 p} Q^{\prime}=0$ and hence

$$
L_{+}\left(x Q^{\prime}\right)=s(-\Delta)^{s} Q .
$$

Similarly,

$$
L_{+}\left(\frac{s}{p} Q\right)=\frac{1}{2}(-\Delta)^{s} \frac{s}{p} Q+\frac{s}{p} Q-(2 p+1) Q^{2 p} \frac{s}{p} Q=\frac{s}{p}\left(-2 p Q^{2 p} Q\right)=-2 s Q^{2 p+1} .
$$

Putting together (3.2) and (3.3) we see that

$$
L_{+}\left(x Q^{\prime}+\frac{s}{p} Q\right)=-2 s Q .
$$

As a consequence,

$$
L_{+} u=\mu Q=L_{+}\left(-\frac{\mu}{2 s}\left(x Q^{\prime}+\frac{s}{p} Q\right)\right) .
$$

But $Q$ is a non degenerate ground state, namely $\operatorname{ker} L_{+}=\operatorname{span}\left\{Q^{\prime}\right\}$, and there is $\vartheta \in \mathbb{R}$ with

$$
u+\frac{\mu}{2 s}\left(x Q^{\prime}+\frac{s}{p} Q\right)=\vartheta Q^{\prime} .
$$

We claim that $\vartheta=0$. Indeed,

$$
u=-\frac{\mu}{2 s}\left(x Q^{\prime}+\frac{s}{p} Q\right)+\vartheta Q^{\prime}
$$

and multiplying by $(2 p+1) Q^{2 p}$ we get

$$
(2 p+1) Q^{2 p} u=-\frac{\mu}{2 s}(2 p+1) Q^{2 p} x Q^{\prime}-\frac{\mu}{2 p}(2 p+1) Q^{2 p}+(2 p+1) \vartheta Q^{2 p} Q^{\prime} .
$$

Since $u \in \mathcal{V}_{0}$,

$$
\left\langle(2 p+1) Q^{2 p} u, Q^{\prime}\right\rangle_{2}=\left\langle u,(2 p+1) Q^{2 p} Q^{\prime}\right\rangle_{2}=0 .
$$

Since $Q$ is an even function, $Q^{\prime}$ is an odd function, and this implies

$$
\begin{aligned}
\left\langle H(Q) Q, Q^{\prime}\right\rangle_{2} & =(2 p+1) \int Q^{2 p+1} Q^{\prime}=0 \\
\left\langle H(Q) Q^{\prime}, Q^{\prime}\right\rangle_{2} & =(2 p+1) \int Q^{2 p} x\left(Q^{\prime}\right)^{2}=0 .
\end{aligned}
$$

On the other hand,

$$
\left\langle H(Q) \vartheta Q^{\prime}, Q^{\prime}\right\rangle_{2}=(2 p+1) \vartheta \int Q^{2 p}\left(Q^{\prime}\right)^{2}>0,
$$

and we conclude that $\vartheta=0$. hence

$$
u=-\frac{\mu}{2 s}\left(x Q^{\prime}+\frac{s}{p} Q\right)
$$


and

$$
0=\int u Q=-\frac{\mu}{2 s} \int x Q Q^{\prime}-\frac{\mu}{2 p} \int Q^{2} .
$$

It is readily seen that $\mu \neq 0$. Moreover, an integration by parts shows that

$$
\int x Q Q^{\prime}=-\frac{1}{2} \int Q^{2}
$$

and thus

$$
\left(\frac{1}{2 p}-\frac{1}{4 s}\right) \int Q^{2}=0
$$

Since $p<2 s$, we deduce $Q=0$, which is clearly impossible. The proof is complete.

Remark 3.2. Actually the previous proof yields a positive constant $\alpha_{0}$ such that

$$
\left\langle L_{+}(v), v\right\rangle \geq \alpha_{0}\|v\|_{2}^{2} \quad \text { for every } v \in \mathcal{V}_{0} .
$$

Hence $\mathcal{V}_{0}$ becomes a complete normed space with respect to the norm $v \mapsto \sqrt{\left\langle L_{+} v, v\right\rangle}$. Now the Closed Graph Theorem tells us that, for a suitable $\bar{\alpha}>0$,

$$
\left\langle L_{+}(v), v\right\rangle \geq \bar{\alpha}\|v\|_{H^{s}}^{2} \quad \text { for every } v \in \mathcal{V}_{0} .
$$

Lemma 3.3. Suppose $\phi \in L^{2}\left(\mathbb{R}^{N}, \mathbb{C}\right)$ satisfies $\|\phi\|_{2}=\|Q\|_{2}$. Then

$$
\langle Q, \mathfrak{R e}(\phi-Q)\rangle_{2}=-\frac{1}{2}\left(\|\mathfrak{R e}(\phi-Q)\|_{2}^{2}+\|\mathfrak{I m}(\phi-Q)\|_{2}^{2}\right)=-\frac{1}{2}\|\phi-Q\|_{2}^{2} .
$$

Proof. It follows from a direct computation and the fact that $Q$ is real-valued.

Proposition 3.4. Assume

$$
0<s<1, \quad 1<p<\frac{2 s}{N} .
$$

Let us take $\phi$ as in (3.5), such that

$$
\left\langle\mathfrak{R e}(\phi-Q), H(Q) \frac{\partial Q}{\partial x_{j}}\right\rangle_{2}=0 \quad \text { for } j=1,2, \ldots, N .
$$

Then

$$
\left\langle L_{+}(\mathfrak{R e}(\phi-Q)), \mathfrak{R e}(\phi-Q)\right\rangle \geq C\|\mathfrak{R e}(\phi-Q)\|_{H^{s}}^{2}-C_{1}\|\phi-Q\|_{H^{s}}^{4}-C_{2}\|\phi-Q\|_{H^{s}}^{3}
$$

for suitable constants $C, C_{1}, C_{2}>0$.

Proof. It is not restrictive to fix $\|Q\|_{2}=1$. We decompose $U=\mathfrak{R e}(\phi-Q)$ as $U=U_{\|}+U_{\perp}$, where $U_{\|}=\langle U, Q\rangle_{2} Q$. By formula (3.5), we get

$$
\left\|(-\Delta)^{\frac{s}{2}} U\right\|_{2}^{2} \leq 2\left\|(-\Delta)^{\frac{s}{2}} U_{\|}\right\|_{2}^{2}+2\left\|(-\Delta)^{\frac{s}{2}} U_{\perp}\right\|_{2}^{2}=\frac{1}{2}\|\phi-U\|_{2}^{4}\left\|(-\Delta)^{\frac{s}{2}} Q\right\|_{2}^{2}+2\left\|(-\Delta)^{\frac{s}{2}} U_{\perp}\right\|_{2}^{2},
$$

so that

$$
\left\|(-\Delta)^{\frac{s}{2}} U_{\perp}\right\|_{2}^{2} \geq \frac{1}{2}\left\|(-\Delta)^{\frac{s}{2}} U\right\|_{2}^{2}-\frac{1}{4}\|\phi-Q\|_{2}^{4}\left\|(-\Delta)^{\frac{s}{2}} Q\right\|_{2}^{2} .
$$

The symmetry of $L_{+}$implies

$$
\left\langle L_{+} U, U\right\rangle=\left\langle L_{+} U_{\|}, U_{\|}\right\rangle+2\left\langle L_{+} U_{\perp}, U_{\|}\right\rangle+\left\langle L_{+} U_{\perp}, U_{\perp}\right\rangle .
$$

But $\left\langle U_{\|}, H(Q) \partial Q / \partial x_{j}\right\rangle_{2}=0$, hence also $\left\langle U_{\perp}, H(Q) \partial Q / \partial x_{j}\right\rangle_{2}=0$ by (3.6). As a consequence, $U_{\perp} \in \mathcal{V}_{0}$. We deduce from (3.4), (3.5) and (3.8) that

$$
\left\langle L_{+} U_{\perp}, U_{\perp}\right\rangle \geq C\left(\|U\|_{H^{s}}^{2}-\|\phi-Q\|_{2}^{4}\right)
$$


Again, from (3.5), we get

$$
\begin{aligned}
\left\langle L_{+} U_{\perp}, U_{\|}\right\rangle & =\langle Q, U\rangle_{2}\left\langle L_{+} U_{\perp}, Q\right\rangle=-\frac{1}{2}\|\phi-Q\|_{2}^{2}\left\langle U_{\perp}, L_{+} Q\right\rangle \\
& =\frac{p}{2}\|\phi-Q\|_{2}^{2}\left(\mathfrak{R e} \int(-\Delta)^{s / 2} U(-\Delta)^{s / 2} Q-\langle U, Q\rangle_{2}\left\|(-\Delta)^{s / 2} Q\right\|_{2}^{2}\right) \\
& \geq-\frac{p}{2}\|\phi-Q\|_{2}^{2}\left\|(-\Delta)^{s / 2}(\phi-Q)\right\|_{2}\left\|(-\Delta)^{s / 2} Q\right\|_{2} \geq-C\|\phi-Q\|_{H^{s}}^{3} .
\end{aligned}
$$

Finally, we get

$$
\left\langle L_{+} U_{\|}, U_{\|}\right\rangle=\langle U, Q\rangle_{2}^{2}\left\langle L_{+} Q, Q\right\rangle=\frac{1}{4}\|\phi-Q\|_{2}^{4}\left\langle L_{+} Q, Q\right\rangle=-\frac{p}{2}\|Q\|_{H^{s}}^{2}\|\phi-Q\|_{2}^{4} .
$$

Putting together (3.9), (3.10), (3.11) and (3.12), we complete the proof.

Let us denote by $L_{-}$the imaginary part of the linearized operator at $Q$, namely

$$
L_{-}=\frac{1}{2}(-\Delta)^{s}+1-Q^{2 p}
$$

Proposition 3.5. There results

$$
\inf _{\substack{v \neq 0 \\\langle v, Q\rangle_{H^{s}}=0}} \frac{\left\langle L_{-} v, v\right\rangle}{\|v\|_{H^{s}}^{2}}>0 .
$$

Proof. It suffices to prove that

$$
\inf _{\substack{v \neq 0 \\ v \neq 0}} \frac{\left\langle L_{-} v, v\right\rangle}{\|v\|_{2}^{2}}>0 .
$$

First of all, let us recall that $\lim _{|x| \rightarrow+\infty} Q(x)=0$. Since, as claimed in [11, Section 3.2],

$$
\sigma_{\text {ess }}\left(\frac{1}{2}(-\Delta)^{s}+1\right)=[1,+\infty)
$$

and since the multiplication operator by $Q^{2 p}$ is compact, we deduce that

$$
\sigma_{\text {ess }}\left(L_{-}\right)=[1,+\infty)
$$

It now follows that $L_{-}$has a discrete spectrum over $(-\infty, 1)$ which consists of eigenvalues of finite multiplicity. Of course $Q \in \operatorname{ker} L_{-}$, so that 0 is an eigenvalue of $L_{-}$and $Q$ is an associated eigenfunction. But $Q$ never changes sign, and we deduce from the proof of Lemma 8.2 in [12] that 0 is the smallest eigenvalue of $L_{-}$. In particular, $L_{-}$is a non-negative operator. Once it is proved [12] that the heat semigroup $\mathcal{H}_{s}(t)=\exp \left\{-t(-\Delta)^{s}\right\}$ is positivity preserving, namely its kernel is a positive function, standard arguments (see [26, Section 10.5] or [27, Theorems 10.32 and 10.33]) show now that this eigenvalue is simple. Therefore, $\operatorname{ker} L_{-}=\operatorname{span} Q$. Let us set

$$
\omega=\inf \left\{\left\langle L_{-} v, v\right\rangle \mid\|v\|_{2}=1,\langle v, Q\rangle_{H^{s}}=0\right\},
$$

and assume for the sake of contradiction that $\omega=0$. If $\left\{v_{n}\right\}_{n}$ is a minimizing sequence for $\omega$, it follows from the regularity properties of $Q$ that $\left\{v_{n}\right\}_{n}$ is bounded in $H^{s}(\mathbb{R}, \mathbb{C})$, and we can assume without loss of generality that this sequence converges weakly to some $v$; as a consequence, $\langle v, Q\rangle_{H^{s}}=0$. Again, the compactness of the multiplication operator by $Q^{2 p}$ entails

$$
0 \leq\left\langle L_{-} v, v\right\rangle \leq \liminf _{n \rightarrow+\infty}\left(\left\|v_{n}\right\|_{H^{s}}^{2}-\int Q^{2 p} v_{n}^{2}\right)=\lim _{n \rightarrow+\infty}\left\langle L_{-} v_{n}, v_{n}\right\rangle=0
$$

and thus $\left\langle L_{-} v, v\right\rangle=0$. But then

$$
\begin{aligned}
\|v\|_{H^{s}}^{2} \leq \liminf _{n \rightarrow+\infty}\left\|v_{n}\right\|_{H^{s}}^{2} & \leq \limsup _{n \rightarrow+\infty}\left\|v_{n}\right\|_{H^{s}}^{2}=\lim _{n \rightarrow+\infty}\left(\left\langle L_{-} v_{n}, v_{n}\right\rangle+\int Q^{2 p} v_{n}^{2}\right) \\
& =\left\langle L_{-} v, v\right\rangle+\int Q^{2 p} v^{2} \leq\|v\|_{H^{s}}^{2} .
\end{aligned}
$$


We have proved that $v_{n} \rightarrow v$ strongly, and that $v$ solves the minimization problem for $\omega$. Therefore, $\lambda$ and $\mu$ being two Lagrange multipliers, we have that

$$
\left\langle L_{-} v, \eta\right\rangle=\lambda\langle v, \eta\rangle_{2}+\mu\langle Q, \eta\rangle_{H^{s}}
$$

for every $\eta \in H^{s}(\mathbb{R}, \mathbb{C})$. Choosing $\eta=v$ yields $\lambda=0$; choosing $\eta=Q$ and recalling that $L_{-} Q=0$ yields $0=\left\langle v, L_{-} Q\right\rangle=\left\langle L_{-} v, Q\right\rangle=\mu\|Q\|_{H^{s}}^{2}$. Hence $\mu=0$, and we conclude that $L_{-} v=0$. Since we know that $\operatorname{ker} L_{-}=\operatorname{span} Q$, for some $\theta \in \mathbb{R}$ we must have $v=\theta Q$. But then $0=\theta\|Q\|_{H^{s}}^{2}$, a contradiction. This shows that $\omega>0$, namely the validity of (3.13).

Lemma 3.6. Fix $\phi \in H^{s}\left(\mathbb{R}^{N}, \mathbb{C}\right)$ such that $\|\phi\|_{2}=\|Q\|_{2}$ and

$$
\inf _{\substack{x \in \mathbb{R}^{N} \\ \vartheta \in[0,2 \pi)}}\left\|\phi-e^{\mathrm{i} \vartheta} Q(\cdot-x)\right\|_{H^{s}} \leq\|Q\|_{H^{s}} .
$$

Then

$$
\inf _{\substack{x \in \mathbb{R}^{N} \\ \vartheta \in[0,2 \pi)}}\left\|\phi-e^{\mathrm{i} \vartheta} Q(\cdot-x)\right\|_{H^{s}}^{2}
$$

is achieved at some $x_{0} \in \mathbb{R}^{N}$ and $\vartheta_{0} \in[0,2 \pi)$. Moreover, writing $\phi\left(\cdot+x_{0}\right) e^{-\mathrm{i} \vartheta_{0}}=Q+W$ where $W=U+\mathrm{i} V$, we have the relations, for $j=1,2, \ldots, N$ :

$$
\left\langle U, H(Q) \frac{\partial Q}{\partial x_{j}}\right\rangle_{2}=0 \quad \text { and } \quad\langle V, Q\rangle_{H^{s}}=0 .
$$

Proof. The variable $\vartheta \in[0,2 \pi)$ is clearly harmless, since $e^{\mathrm{i} \vartheta}$ describes the compact circle $S^{1} \subset$ $\mathbb{C}$. We can therefore assume that $\vartheta=0$. Consider the auxiliary function $\mathfrak{n}: \mathbb{R}^{N} \rightarrow \mathbb{R}$ defined by setting $\mathfrak{n}(x)=\|\phi-Q(\cdot-x)\|_{H^{s}}^{2}$. Plainly, $\mathfrak{n}$ is a continuous function, and

$$
\begin{aligned}
\mathfrak{n}(x) & =2\|Q\|_{2}^{2}+\left\|(-\Delta)^{\frac{s}{2}} Q\right\|_{2}^{2}+\left\|(-\Delta)^{\frac{s}{2}} \phi\right\|_{2}^{2} \\
& -2 \mathfrak{R e} \int_{\mathbb{R}^{N}} \overline{\phi(y)} Q(y-x) d y-\mathfrak{R e} \int \overline{(-\Delta)^{\frac{s}{2}} \phi(y)}(-\Delta)^{\frac{s}{2}} Q(y) d y
\end{aligned}
$$

because $\|\phi\|_{2}=\|Q\|_{2}$. Since both $Q(\cdot-x)$ and $(-\Delta)^{\frac{s}{2}} Q(\cdot-x)$ decay to zero as $|x| \rightarrow+\infty$ (thanks to Theorem 2.2 and using the equation satisfied by $Q$ ), we deduce that they also converge weakly to zero as $|x| \rightarrow+\infty$. It easily follows that

$$
\lim _{|x| \rightarrow+\infty} \mathfrak{n}(x)=2\|Q\|_{2}^{2}+\left\|(-\Delta)^{\frac{s}{2}} Q\right\|_{2}^{2}+\left\|(-\Delta)^{\frac{s}{2}} \phi\right\|_{2}^{2}>\|Q\|_{H^{s}}^{2} .
$$

On the other hand, assumption (3.14) entails that, for every $\delta>0$, there exists a point $x_{\delta} \in \mathbb{R}$ with $\mathfrak{n}\left(x_{\delta}\right) \leq\|Q\|_{H^{s}}^{2}+\delta$. As a consequence, the function $\mathfrak{n}$ attains its infimum on some ball $B(0, R)$, for a suitable $R>0$, and the proof is complete. Finally, we compute the EulerLagrange equations associated to the variational problem (3.15) by differentiating with respect to $\theta$ and to $x_{j}$ :

$$
\begin{aligned}
& \left\langle\phi-e^{\mathrm{i} \vartheta_{0}} Q\left(\cdot-x_{0}\right),-\mathrm{i} e^{\mathrm{i} \vartheta_{0}} Q\left(\cdot-x_{0}\right)\right\rangle_{H^{s}}=0 \\
& \left\langle\phi-e^{\mathrm{i} \vartheta_{0}} Q\left(\cdot-x_{0}\right),-e^{\mathrm{i} \vartheta_{0}} \frac{\partial Q}{\partial x_{j}}\left(\cdot-x_{0}\right)\right\rangle_{H^{s}}=0 .
\end{aligned}
$$

Equation (3.17) yields

$$
\begin{aligned}
\mathfrak{R e} \int\left(\phi-e^{\mathrm{i} \vartheta_{0}} Q\left(\cdot-x_{0}\right)\right) & \overline{-\mathrm{i} e^{\mathrm{i} \vartheta_{0}} Q\left(\cdot-x_{0}\right)} \\
+ & \frac{1}{2} \mathfrak{R e} \int(-\Delta)^{\frac{s}{2}}\left(\phi-e^{\mathrm{i} \vartheta_{0}} Q\left(\cdot-x_{0}\right)\right) \overline{(-\Delta)^{\frac{s}{s}}\left(-\mathrm{i} e^{\mathrm{i} \vartheta_{0}} Q\left(\cdot-x_{0}\right)\right)}=0,
\end{aligned}
$$

namely

$$
\int Q\left(\cdot-x_{0}\right) \mathfrak{I m}\left(\phi e^{-\mathrm{i} \vartheta_{0}}\right)+\frac{1}{2} \int(-\Delta)^{\frac{s}{2}} Q\left(\cdot-x_{0}\right) \mathfrak{I m}\left((-\Delta)^{\frac{s}{2}}\left(e^{-\mathrm{i} \vartheta_{0}} \phi\right)\right)=0
$$


or $\langle Q, V\rangle_{H^{s}}=0$. Similarly, equation (3.18) yields

$$
\begin{aligned}
\mathfrak{R e} \int\left(\phi-e^{\mathrm{i} \vartheta_{0}} Q(\cdot-\right. & \left.\left.x_{0}\right)\right) \overline{-e^{\mathrm{i} \vartheta_{0}} \frac{\partial Q}{\partial x_{j}}\left(\cdot-x_{0}\right)} \\
& +\frac{1}{2} \mathfrak{R e} \int(-\Delta)^{\frac{s}{2}}\left(\phi-e^{\mathrm{i} \vartheta_{0}} Q\left(\cdot-x_{0}\right)\right) \overline{(-\Delta)^{\frac{s}{2}}\left(-e^{\mathrm{i} \vartheta_{0}} \frac{\partial Q}{\partial x_{j}}\left(\cdot-x_{0}\right)\right)}=0,
\end{aligned}
$$

or

$$
\int U \frac{\partial Q}{\partial x_{j}}+\int Q \frac{\partial Q}{\partial x_{j}}+\frac{1}{2} \int(-\Delta)^{\frac{s}{2}} U(-\Delta)^{\frac{s}{2}} \frac{\partial Q}{\partial x_{j}}+\frac{1}{2} \int(-\Delta)^{\frac{s}{2}} Q(-\Delta)^{\frac{s}{2}} \frac{\partial Q}{\partial x_{j}}=0 .
$$

Since

and using the fact that

$$
\int Q \frac{\partial Q}{\partial x_{j}}=0=\int(-\Delta)^{\frac{s}{2}} Q(-\Delta)^{\frac{s}{2}} \frac{\partial Q}{\partial x_{j}}
$$

$$
\frac{1}{2}(-\Delta)^{s} \frac{\partial Q}{\partial x_{j}}+\frac{\partial Q}{\partial x_{j}}=H(Q) \frac{\partial Q}{\partial x_{j}}
$$

we finally deduce $\left\langle U, H(Q) \frac{\partial Q}{\partial x_{j}}\right\rangle_{2}=0$.

Lemma 3.7. If $p \in(0,1)$, there exists a constant $C>0$ such that

$$
\left.|| z\right|^{p-1} z-|w|^{p-1} w|\leq C| z-\left.p\right|^{p}, \quad \text { for every } z, w \in \mathbb{C} .
$$

Proof. Let $z, w \in \mathbb{C}$ be given and let $\vartheta \in[0,2 \pi)$ be the angle between them. Without loss of generality, we may assume that $t=|z| /|w|>1$. Since we have

$$
\frac{\left.|| z\right|^{p-1} z-|w|^{p-1} w \mid}{|z-w|^{p}} \leq \sup _{\substack{t \in[1, \infty) \\ \vartheta \in[0,2 \pi)}} \frac{\left(t^{2 p}+1-2 t^{p} \cos \vartheta\right)^{1 / 2}}{\left(t^{2}+1-2 t \cos \vartheta\right)^{p / 2}}<+\infty,
$$

the assertion follows.

Proposition 3.8. Let $\Psi(u)=\int|u|^{2 p+2}$. Then $\Psi$ is of class $C^{2}$ on $H^{s}\left(\mathbb{R}^{N}, \mathbb{C}\right)$ for $0<p<\frac{2 s}{N}$.

Proof. Since $\Psi^{\prime \prime}$ is a symmetric bilinear form on the real Hilbert space $H^{s}\left(\mathbb{R}^{N}, \mathbb{C}\right)$, its norm as a bilinear form equals the norm of its associated quadratic form, see for example [8, Lemma 2.1, pag. 173]; therefore we prove that

$$
\lim _{v \rightarrow u} \sup _{h \neq 0} \frac{\Psi^{\prime \prime}(u)(h, h)-\Psi^{\prime \prime}(v)(h, h)}{\|h\|_{H^{s}}^{2}}=0 .
$$

From (2.2) we know that $\Psi^{\prime \prime}(u)$ splits into two terms (we drop some multiplicative constants),

$$
\Psi_{1}^{\prime \prime}(u)(h, h):=\int|u|^{2 p} h \bar{h} \quad \text { and } \quad \Psi_{2}^{\prime \prime}(u)(h, h):=\int|u|^{2 p-2}(\mathfrak{R e}(u \bar{h}))^{2}, \quad h \in H^{s}\left(\mathbb{R}^{N}, \mathbb{C}\right),
$$

which we shall treat separately. Let $\left\{u_{n}\right\}_{n} \subset H^{s}\left(\mathbb{R}^{N}, \mathbb{C}\right)$ be such that $u_{n} \rightarrow u$ strongly as $n \rightarrow \infty$. Then, in the case $2 p \leq 1$, by the Hölderianity of the map $s \mapsto s^{2 p}$ we obtain that

$$
\left|\Psi_{1}^{\prime \prime}\left(u_{n}\right)(h, h)-\Psi_{1}^{\prime \prime}(u)(h, h)\right| \leq\left. C \int|| u_{n}\right|^{2 p}-\left.|u|^{2 p}|| h\right|^{2} \leq C \int\left|u_{n}-u\right|^{2 p}|h|^{2} .
$$

By applying the Hölder inequality with admissible exponents $(q, r)$ respectively,

$$
q:=\frac{N}{p(N-2 s)}>1, \quad r:=\frac{N}{2 p s+(1-p) N} \in\left(1, \frac{N}{N-2 s}\right),
$$

it follows for every $h \in H^{s}\left(\mathbb{R}^{N}, \mathbb{C}\right)$ with $\|h\|_{H^{s}} \leq 1$

$$
\left|\Psi_{1}^{\prime \prime}\left(u_{n}\right)(h, h)-\Psi_{1}^{\prime \prime}(u)(h, h)\right| \leq C\left\|u_{n}-u\right\|_{\frac{2 N}{N-2 s}}^{2 p}\|h\|_{2 r}^{2} \leq C\left\|u_{n}-u\right\|_{\frac{2 N}{N-2 s}}^{2 p},
$$


since $\|h\|_{2 r} \leq C\|h\|_{H^{s}} \leq C$, concluding the proof for $\Psi_{1}^{\prime \prime}$. The opposite case $2 p>1$ can be treated similarly. Let us now come to the treatment of $\Psi_{2}^{\prime \prime}$. We notice that, for $p<1$, we get

$$
\begin{aligned}
& \left.|| u_{n}\right|^{2 p-2}\left(\mathfrak{R e}\left(u_{n} \bar{h}\right)\right)^{2}-|u|^{2 p-2}(\mathfrak{R e}(u \bar{h}))^{2} \mid \\
& \leq\left. 2|h| \max \left\{\left|u_{n}\right|^{p},|u|^{p}\right\}|| u_{n}\right|^{p-1} \mathfrak{R e}\left(u_{n} \bar{h}\right)-|u|^{p-1} \mathfrak{R e}(u \bar{h}) \mid \\
& \leq C \max \left\{\left|u_{n}\right|^{p},|u|^{p}\right\}\left|u_{n}-u\right|^{p}|h|^{2},
\end{aligned}
$$

where we used Lemma 3.7. Now we can proceed as before and conclude the proof.

3.1. Proof of Theorem 1.1. We consider the action $I(\phi)=\frac{1}{2} \mathscr{E}(\phi)+\frac{1}{2}\|\phi\|_{2}^{2}$ and we control the norm of $w$ in terms of the difference $I(\phi)-I(Q)$. Using the scale invariance of $I$, recalling that $\left\langle I^{\prime}(Q), w\right\rangle=0$, the orthogonality conditions (3.16), Propositions 3.4 and 3.5, and taking into account Proposition 3.8, by virtue of Taylor formula, we have

$$
\begin{aligned}
I(\phi)-I(Q) & =I(Q+w)-I(Q)=\left\langle I^{\prime}(Q), w\right\rangle+\frac{1}{2}\left\langle I^{\prime \prime}(Q) w, w\right\rangle+o\left(\|w\|_{H^{s}}^{2}\right) \\
& =\left\langle L_{+} u, u\right\rangle+\left\langle L_{-} v, v\right\rangle+o\left(\|w\|_{H^{s}}^{2}\right) \\
& \geq C\|u\|_{H^{s}}^{2}+C\|v\|_{H^{s}}^{2}+o\left(\|w\|_{H^{s}}^{2}\right)=C\|w\|_{H^{s}}^{2}+o\left(\|w\|_{H^{s}}^{2}\right) .
\end{aligned}
$$

To complete the proof of Theorem 1.1, we observe that for every $\varepsilon>0$ there exists $\delta>0$ such that, if $\phi \in H^{s}\left(\mathbb{R}^{N}, \mathbb{C}\right),\|\phi\|_{2}=\|Q\|_{2}$ and $\mathcal{E}(\phi)-\mathcal{E}(Q)<\delta$, then

$$
\inf _{x \in \mathbb{R}^{N}, \vartheta \in[0,2 \pi)}\left\|\phi-e^{\mathrm{i} \vartheta} Q(\cdot-x)\right\|_{H^{s}}<\varepsilon .
$$

Then, choosing $\mathcal{E}(\phi)-\mathcal{E}(Q)$ small enough, Theorem 1.1 follows. By the uniqueness of solutions to $\min \left\{\mathcal{E}(q): q \in H^{s}\left(\mathbb{R}^{N}, \mathbb{C}\right.\right.$ ), $\left.\|q\|_{2}=\|Q\|_{2}\right\}$ (see Corollary 2.9) the above implication follows by Lions' concentration compactness principle as in [5].

\section{Dynamics of the Ground state}

We first recall the following (cf. [9, Lemma 2.4]).

Lemma 4.1. Let $s, \bar{\sigma} \in(0,1]$ and $\delta>2|\bar{\sigma}-s|$. Then, for any $\varphi \in H^{2(\bar{\sigma}+\delta)}\left(\mathbb{R}^{N}\right)$,

$$
\left\|(-\Delta)^{\bar{\sigma}} \varphi-(-\Delta)^{s} \varphi\right\|_{2} \leq C(\bar{\sigma}, \delta)|\bar{\sigma}-s|\|\varphi\|_{H^{2(\bar{\sigma}+\delta)}},
$$

for a suitable $C(\bar{\sigma}, \delta)>0$ of the form $C(\bar{\sigma}, \delta)=\frac{C_{1}}{\bar{\sigma}}+\frac{C_{2}}{\delta}$ with $C_{1}, C_{2}$ independent of $\bar{\sigma}, \delta$.

Let now $u^{\varepsilon}$ be a solution of the Cauchy problem (1.1). The energy is defined as

$$
E_{\varepsilon}(t)=\frac{1}{2 \varepsilon^{N-2 s}} \int\left|(-\Delta)^{\frac{s}{2}} u^{\varepsilon}(t, x)\right|^{2}+\frac{1}{\varepsilon^{N}} \int V(x)\left|u^{\varepsilon}(t, x)\right|^{2}-\frac{1}{(p+1) \varepsilon^{N}} \int\left|u^{\varepsilon}(t, x)\right|^{2 p+2},
$$

and $E_{\varepsilon}(t)=E_{\varepsilon}(0)$ for every $t \geq 0$. Moreover the mass conservation reads as

Let us set

$$
\frac{1}{\varepsilon^{N}} \int\left|u^{\varepsilon}(t, x)\right|^{2}=\|Q\|_{2}^{2}=: m, \quad t \geq 0, \varepsilon>0 .
$$

$$
\mathbb{J}_{s}:=-C(N, s) \iint \frac{Q(x)(Q(x)-Q(x-z))\left(1-\cos \left\langle z, v_{0}\right\rangle\right)}{|z|^{N+2 s}} d x d z,
$$

and define

$$
\mathcal{H}(t):=\frac{1}{2} m|v(t)|^{2 s}+m V(x(t)), \quad t \geq 0 .
$$

Then we have the following

Lemma 4.2. For $t \in[0, \infty)$ and $\varepsilon>0$ we have

$$
E_{\varepsilon}(t)=\mathcal{E}(Q)+\mathcal{H}(t)+\mathcal{O}\left(\varepsilon^{2}\right)+\frac{1}{2} \mathbb{J}_{s} .
$$

Moreover, $\mathbb{J}_{s}=\mathcal{O}(1-s)$. 
Proof. Assuming $x_{0}=0$ for simplicity, we observe that

$$
\frac{1}{\varepsilon^{N-2 s}} \iint \frac{\left|Q\left(\frac{x}{\varepsilon}\right) e^{\frac{\mathrm{i}}{\varepsilon}\left\langle x, v_{0}\right\rangle}-Q\left(\frac{y}{\varepsilon}\right) e^{\frac{\mathrm{i}}{\varepsilon}\left\langle y, v_{0}\right\rangle}\right|^{2}}{|x-y|^{N+2 s}} d x d y=\iint \frac{\left|Q(x) e^{\mathrm{i}\left\langle x, v_{0}\right\rangle}-Q(y) e^{\mathrm{i}\left\langle y, v_{0}\right\rangle}\right|^{2}}{|x-y|^{N+2 s}} d x d y .
$$

Recalling the identity [7, formula (3.12)]

$$
\int \frac{1-\cos \left\langle z, v_{0}\right\rangle}{|z|^{N+2 s}} d z=\frac{\left|v_{0}\right|^{2 s}}{C(N, s)}
$$

we obtain, on account of [7, Proposition 3.4], the following conclusion

$$
\begin{aligned}
& \iint \frac{\left|Q(x) e^{\mathrm{i}\left\langle x, v_{0}\right\rangle}-Q(y) e^{\mathrm{i}\left\langle y, v_{0}\right\rangle}\right|^{2}}{|x-y|^{N+2 s}} d x d y \\
& =\iint \frac{\left|Q(x) e^{\mathrm{i}\left\langle x, v_{0}\right\rangle}-Q(x) e^{\mathrm{i}\left\langle y, v_{0}\right\rangle}+Q(x) e^{\mathrm{i}\left\langle y, v_{0}\right\rangle}-Q(y) e^{\mathrm{i}\left\langle y, v_{0}\right\rangle}\right|^{2}}{|x-y|^{N+2 s}} d x d y \\
& =\iint \frac{|Q(x)-Q(y)|^{2}}{|x-y|^{N+2 s}} d x d y+\iint \frac{|Q(x)|^{2}\left|e^{\mathrm{i}\left\langle x, v_{0}\right\rangle}-e^{\mathrm{i}\left\langle y, v_{0}\right\rangle}\right|^{2}}{|x-y|^{N+2 s}} d x d y+\frac{2}{C(N, s)} \mathbb{J}_{s} \\
& =\frac{2}{C(N, s)}\left\|(-\Delta)^{\frac{s}{2}} Q\right\|_{2}^{2}+2 \iint \frac{|Q(x)|^{2}\left(1-\cos \left\langle x-y, v_{0}\right\rangle\right)}{|x-y|^{N+2 s}} d x d y+\frac{2}{C(N, s)} \mathbb{J}_{s} \\
& =\frac{2}{C(N, s)}\left\|(-\Delta)^{\frac{s}{2}} Q\right\|_{2}^{2}+2 \iint \frac{|Q(x)|^{2}\left(1-\cos \left\langle z, v_{0}\right\rangle\right)}{|z|^{N+2 s}} d x d z+\frac{2}{C(N, s)} \mathbb{J}_{s} \\
& =\frac{2}{C(N, s)}\left\|(-\Delta)^{\frac{s}{2}} Q\right\|_{2}^{2}+2 \int|Q(x)|^{2} \frac{\left|v_{0}\right|^{2 s}}{C(N, s)}+\frac{2}{C(N, s)} \mathbb{J}_{s} \\
& =\frac{2}{C(N, s)}\left(\left\|(-\Delta)^{\frac{s}{2}} Q\right\|_{2}^{2}+\left|v_{0}\right|^{2 s}\|Q\|_{2}^{2}+\mathbb{J}_{s}\right) .
\end{aligned}
$$

Therefore,

$$
\left\|(-\Delta)^{\frac{s}{2}}\left(Q(\cdot) e^{\mathrm{i}\left\langle\cdot, v_{0}\right\rangle}\right)\right\|_{2}^{2}=\left\|(-\Delta)^{\frac{s}{2}} Q\right\|_{2}^{2}+\left|v_{0}\right|^{2 s}\|Q\|_{2}^{2}+\mathbb{J}_{s} .
$$

We know from a direct elementary computation (since $\left\|(-\Delta)^{1 / 2} \varphi\right\|_{2}=\|\nabla \varphi\|_{2}$ ) that

$$
\left\|(-\Delta)^{1 / 2}\left(Q(\cdot) e^{\mathrm{i}\left\langle\cdot \cdot v_{0}\right\rangle}\right)\right\|_{2}^{2}=\left\|(-\Delta)^{1 / 2} Q\right\|_{2}^{2}+\left|v_{0}\right|^{2}\|Q\|_{2}^{2} .
$$

From Lemma 4.1, we learn that

$$
\begin{aligned}
\left\|(-\Delta)^{\frac{s}{2}}\left(Q(\cdot) e^{\mathrm{i}\left\langle\cdot v_{0}\right\rangle}\right)\right\|_{2}^{2} & =\left\|(-\Delta)^{1 / 2}\left(Q(\cdot) e^{\mathrm{i}\left\langle\cdot, v_{0}\right\rangle}\right)\right\|_{2}^{2}+\mathcal{O}\left((1-s)^{2}\right), \\
\left\|(-\Delta)^{\frac{s}{2}} Q\right\|_{2}^{2} & =\left\|(-\Delta)^{1 / 2} Q\right\|_{2}^{2}+\mathcal{O}\left((1-s)^{2}\right),
\end{aligned}
$$

Taking into account that $\left|v_{0}\right|^{2 s}-\left|v_{0}\right|^{2}=\mathcal{O}(1-s)$, it follows by comparing (4.2) and (4.3) that $\mathbb{J}_{s}=\mathcal{O}(1-s)$. Whence, by energy conservation, we conclude that

$$
\begin{aligned}
E_{\varepsilon}(t)=E_{\varepsilon}(0) & =\frac{1}{2}\left\|(-\Delta)^{\frac{s}{2}} Q\right\|_{2}^{2}+\frac{1}{2}\left|v_{0}\right|^{2 s}\|Q\|_{2}^{2}+\int V(\varepsilon x)|Q(x)|^{2}-\frac{1}{p+1} \int|Q|^{2 p+2}+\frac{1}{2} \mathbb{J}_{s} \\
=\mathcal{E}(Q)+\frac{1}{2} m\left|v_{0}\right|^{2 s}+ & m V(0)-m V(0)+\int V(\varepsilon x)|Q(x)|^{2}+\frac{1}{2} \mathbb{J}_{s} \\
& =\mathcal{E}(Q)+\mathcal{H}(t)+\int V(\varepsilon x)|Q(x)|^{2} d x-m V(0)+\frac{1}{2} \mathbb{J}_{s} .
\end{aligned}
$$

It is readily checked that $\mathcal{H}$ is conserved along the trajectory $x(t)$, in light of equation (1.3). Since the Hessian $\nabla^{2} V$ is bounded and, by the radial symmetry of $Q$,

$$
\int\langle x, \nabla V(0)\rangle|Q(x)|^{2}=0
$$

we conclude that $\int V(\varepsilon x)|Q(x)|^{2}-m V(0)=\mathcal{O}\left(\varepsilon^{2}\right)$. This ends the proof. 
Remark 4.3. Unlike the local case $s=1$, in the cases $s \in(0,1)$ we cannot expect a precise conclusion as $E_{\varepsilon}(t)=\mathcal{E}(r)+\mathcal{H}(t)+O\left(\varepsilon^{2}\right)$. Indeed, the fractional Laplacian does not obey a Leibniz rule for differentiating products.

For the fractional norms of $u^{\varepsilon}$, we have the following

Lemma 4.4. There exists a constant $C>0$ such that

$$
\left\|(-\Delta)^{\frac{s}{2}} u^{\varepsilon}(t)\right\|_{2} \leq C \varepsilon^{\frac{N-2 s}{2}},
$$

for every $t \geq 0$ and every $\varepsilon>0$.

Proof. Since $V$ is bounded from below and $E_{\varepsilon}(t)$ is uniformly bounded with respect to $t \geq 0$, $\varepsilon>0$ and $s \in(0,1]$ by Lemma 4.2, we deduce that, for all $t \geq 0$,

$$
\begin{aligned}
\left\|(-\Delta)^{\frac{s}{2}} u^{\varepsilon}(t)\right\|_{2}^{2} \leq C \varepsilon^{N-2 s}+C \varepsilon^{-2 s} \int\left|u^{\varepsilon}(t)\right|^{2 p+2} & \\
& \leq C\left(\varepsilon^{N-2 s}+\varepsilon^{-2 s}\left\|u^{\varepsilon}(t)\right\|_{2}^{2 p+2-\frac{N p}{s}}\left\|(-\Delta)^{\frac{s}{2}} u^{\varepsilon}(t)\right\|_{2}^{\frac{N p}{s}}\right) .
\end{aligned}
$$

Here we have used the Sobolev-Gagliardo-Nirenberg inequality (1.7) with exponent

$$
\alpha:=\frac{2 s(p+1)-N p}{2 s(p+1)} \in(0,1) .
$$

Recalling that $\left\|u^{\varepsilon}(t)\right\|_{2}=\sqrt{m} \varepsilon^{N / 2}$ by the conservation of the mass, we can write (4.4) as

$$
\left\|(-\Delta)^{\frac{s}{2}} u^{\varepsilon}(t)\right\|_{2}^{2} \leq C\left(\varepsilon^{N-2 s}+\varepsilon^{-2 s} \varepsilon^{\frac{N}{2}\left(2 p+2-\frac{N p}{s}\right)}\left\|(-\Delta)^{\frac{s}{2}} u^{\varepsilon}(t)\right\|_{2}^{\frac{N p}{s}}\right) .
$$

Now, setting for simplicity $\mathscr{N}=\mathscr{N}(\varepsilon)=\left\|(-\Delta)^{\frac{s}{2}} u^{\varepsilon}(t)\right\|_{2}>0$, (4.5) becomes

$$
\mathscr{N}^{2} \leq C\left(\varepsilon^{N-2 s}+\varepsilon^{-2 s} \varepsilon^{\frac{N}{2}\left(2 p+2-\frac{N p}{s}\right)} \mathscr{N}^{\frac{N p}{s}}\right) .
$$

We claim that $\mathscr{N} \leq C \varepsilon^{\frac{N-2 s}{2}}$. Indeed, we rescale $\mathscr{N}=\varepsilon^{\frac{N-2 s}{2}} \mathscr{Z}$ and deduce that

$$
\mathscr{Z}^{2} \leq C\left(1+\mathscr{Z}^{\frac{N p}{s}}\right) .
$$

Since $N p<2 s$ by assumption, we are lead to $\mathscr{Z} \leq C$ and the proof is complete.

Define now

$$
\Psi^{\varepsilon}(t, x):=\exp \left(-\frac{\mathrm{i}}{\varepsilon}\langle\varepsilon x+x(t), v(t)\rangle\right) u^{\varepsilon}(\varepsilon x+x(t)), \quad x \in \mathbb{R}^{N}, t \geq 0,
$$

where $(x(t), v(t))$ is the solution to problem (1.3). Notice that the exponential function is a globally Lipschitz continuous complex valued function with modulus equal to one. Then, by a variant of $\left[7\right.$, Lemma 5.3], it follows that $\Psi^{\varepsilon}(t, \cdot) \in H^{s}\left(\mathbb{R}^{N}, \mathbb{C}\right)$ for any $t \geq 0$ and $\varepsilon>0$.

We have the following

Lemma 4.5. We have

$$
\mathcal{E}\left(\Psi^{\varepsilon}(t)\right)=\frac{1}{2} m|v(t)|^{2 s}+\frac{\mathbb{M}(t, \varepsilon, s)}{2}-\frac{1}{\varepsilon^{N}} \int V(x)\left|u^{\varepsilon}(t, x)\right|^{2}+E_{\varepsilon}(t),
$$

for every $t \geq 0$ and every $\varepsilon>0$.

Proof. Proceeding as in the proof of Lemma 4.2, we compute

$\int\left|(-\Delta)^{\frac{s}{2}} \Psi^{\varepsilon}(t)\right|^{2}=\frac{C(N, s)}{2} \iint \frac{\left|\Psi^{\varepsilon}(t, x)-\Psi^{\varepsilon}(t, y)\right|^{2}}{|x-y|^{N+2 s}} d x d y=\mathbb{I}_{1}(t, \varepsilon, s)+\mathbb{I}_{2}(t, \varepsilon, s)+\mathbb{M}(t, \varepsilon, s)$,

where we have set

$$
\begin{aligned}
& \mathbb{I}_{1}(t, \varepsilon, s):=\frac{C(N, s)}{2} \iint \frac{\left|u^{\varepsilon}(t, \varepsilon x+x(t))-u^{\varepsilon}(t, \varepsilon y+x(t))\right|^{2}}{|x-y|^{N+2 s}} d x d y \\
& \mathbb{I}_{2}(t, \varepsilon, s):=\frac{C(N, s)}{2} \iint\left|u^{\varepsilon}(t, \varepsilon x+x(t))\right|^{2} \frac{e^{\frac{i}{\varepsilon}\langle\varepsilon x+x(t), v(t)\rangle}-\left.e^{\frac{i}{\varepsilon}\langle\varepsilon y+x(t), v(t)\rangle}\right|^{2}}{|x-y|^{N+2 s}} d x d y
\end{aligned}
$$


and

$$
\begin{aligned}
& \mathbb{M}(t, \varepsilon, s):= \\
& \quad C(N, s) \iint \mathfrak{R e}\left[u^{\varepsilon}(t, \varepsilon x+x(t)) \overline{\left[u^{\varepsilon}(t, \varepsilon x+x(t))-u^{\varepsilon}(t, \varepsilon y+x(t))\right]} \frac{e^{-i\langle x-y, v(t)\rangle}-1}{|x-y|^{N+2 s}}\right] d x d y .
\end{aligned}
$$

By changing variables, and recalling again (4.1), it readily follows that

$$
\begin{aligned}
& \mathbb{I}_{1}(t, \varepsilon, s)=\varepsilon^{2 s-N}\left\|(-\Delta)^{\frac{s}{2}} u^{\varepsilon}(t)\right\|_{2}^{2}, \\
& \mathbb{I}_{2}(t, \varepsilon, s)=\varepsilon^{-N}|v(t)|^{2 s}\left\|u^{\varepsilon}(t)\right\|_{2}^{2}=m|v(t)|^{2 s} \\
& \mathbb{M}(t, \varepsilon, s)=C(N, s) \varepsilon^{2 s-N} \iint \mathfrak{R e}\left[u^{\varepsilon}(t, x) \overline{\left[u^{\varepsilon}(t, x)-u^{\varepsilon}(t, y)\right]} \frac{e^{-\frac{i}{\varepsilon}\langle x-y, v(t)\rangle}-1}{|x-y|^{N+2 s}}\right] d x d y .
\end{aligned}
$$

It follows that

$$
\begin{aligned}
\mathcal{E}\left(\Psi^{\varepsilon}(t)\right) & =\frac{1}{2} \int\left|(-\Delta)^{\frac{s}{2}} \Psi^{\varepsilon}(t)\right|^{2}-\frac{1}{p+1} \int\left|\Psi^{\varepsilon}(t)\right|^{2 p+2} \\
& =\frac{1}{2} \frac{1}{\varepsilon^{N-2 s}}\left\|(-\Delta)^{\frac{s}{2}} u^{\varepsilon}(t)\right\|_{2}^{2}+\frac{1}{2} m|v(t)|^{2 s}-\frac{1}{(p+1) \varepsilon^{N}}\left\|u^{\varepsilon}(t, x)\right\|_{2 p+2}^{2 p+2}+\frac{\mathbb{M}(t, \varepsilon, s)}{2} \\
& =\frac{1}{2} m|v(t)|^{2 s}+\frac{\mathbb{M}(t, \varepsilon, s)}{2}-\frac{1}{\varepsilon^{N}} \int V(x)\left|u^{\varepsilon}(t, x)\right|^{2}+E_{\varepsilon}(t),
\end{aligned}
$$

concluding the proof.

Finally, we have the following

Corollary 4.6. There holds

$$
\mathcal{E}\left(\Psi^{\varepsilon}(t)\right)-\mathcal{E}(Q)=\mathscr{E}(t, \varepsilon, s)+\mathcal{O}\left(\varepsilon^{2}\right)
$$

where $\mathscr{E}(t, \varepsilon, s)=\mathscr{E}_{1}(t, \varepsilon, s)+\mathscr{E}_{2}(t, \varepsilon, s)$ and

$$
\begin{aligned}
& \mathscr{E}_{1}(t, \varepsilon, s):=m|v(t)|^{2 s}+\frac{\mathbb{M}(t, \varepsilon, s)+\mathbb{J}_{s}}{2} \\
& \mathscr{E}_{2}(t, \varepsilon, s):=m V(x(t))-\frac{1}{\varepsilon^{N}} \int V(x)\left|u^{\varepsilon}(t, x)\right|^{2},
\end{aligned}
$$

for every $t \geq 0$ and every $\varepsilon>0$. Furthermore $\mathscr{E}(0, \varepsilon, s)=\mathcal{O}\left(\varepsilon^{2}\right)$.

Proof. By combining Lemma 4.5 with Lemma 4.2, we find

$$
\begin{aligned}
\mathcal{E}\left(\Psi^{\varepsilon}(t)\right) & =\frac{1}{2} m|v(t)|^{2 s}+\frac{1}{2} \mathbb{M}(t, \varepsilon, s)-\frac{1}{\varepsilon^{N}} \int V(x)\left|u^{\varepsilon}(t, x)\right|^{2}+E_{\varepsilon}(t) \\
& =\frac{1}{2} m|v(t)|^{2 s}+\frac{1}{2} \mathbb{M}(t, \varepsilon, s)-\frac{1}{\varepsilon^{N}} \int V(x)\left|u^{\varepsilon}(t, x)\right|^{2} \\
& +\mathcal{E}(Q)+\frac{1}{2} m|v(t)|^{2 s}+m V(x(t))+\mathcal{O}\left(\varepsilon^{2}\right)+\frac{1}{2} \mathbb{J}_{s} \\
& =m|v(t)|^{2 s}+\frac{\mathbb{M}(t, \varepsilon, s)+\mathbb{J}_{s}}{2}+\mathcal{E}(Q)+m V(x(t))-\frac{1}{\varepsilon^{N}} \int V(x)\left|u^{\varepsilon}(t, x)\right|^{2}+\mathcal{O}\left(\varepsilon^{2}\right) \\
& =\mathscr{E}_{1}(t, \varepsilon, s)+\mathscr{E}_{2}(t, \varepsilon, s)+\mathcal{O}\left(\varepsilon^{2}\right) .
\end{aligned}
$$


Now, since we have $u^{\varepsilon}(0, \varepsilon x+x(0))=Q(x) e^{\frac{i}{\varepsilon}\left\langle\varepsilon x+x_{0}, v_{0}\right\rangle}$, we obtain

$$
\begin{aligned}
\mathscr{E}_{1}(0, \varepsilon, s)= & m\left|v_{0}\right|^{2 s}+\frac{\mathbb{M}(0, \varepsilon, s)}{2} \\
& -\frac{C(N, s)}{2} \iint \frac{Q(x)(Q(x)-Q(x-z))\left(1-\cos \left\langle z, v_{0}\right\rangle\right)}{|z|^{N+2 s}} d x d z \\
= & m\left|v_{0}\right|^{2 s}+\frac{C(N, s)}{2} \mathfrak{R e} \iint Q(x)\left[Q(x)-Q(y) e^{i\left\langle x-y, v_{0}\right\rangle}\right] \frac{e^{-i\left\langle x-y, v_{0}\right\rangle}-1}{|x-y|^{N+2 s}} d x d y \\
- & \frac{C(N, s)}{2} \iint \frac{Q(x)(Q(x)-Q(x-z))\left(1-\cos \left\langle z, v_{0}\right\rangle\right)}{|z|^{N+2 s}} d x d z \\
= & m\left|v_{0}\right|^{2 s}-C(N, s) \int Q^{2}(x) \int \frac{1-\cos \left\langle z, v_{0}\right\rangle}{|z|^{N+2 s}} d x d z=0 .
\end{aligned}
$$

That $\mathscr{E}_{2}(0, \varepsilon, s)=\mathcal{O}\left(\varepsilon^{2}\right)$ is immediately seen.

Remark 4.7. From Corollary 4.6, it seems evident that the quantity

$$
\begin{aligned}
\varepsilon^{2 s-N} \iint \frac{\mathfrak{R e}\left[u^{\varepsilon}(t, x) \overline{\left[\left(u^{\varepsilon}(t, x)-u^{\varepsilon}(t, x-z)\right)\right]}\left(e^{-\frac{i}{\varepsilon}\langle z, v(t)\rangle}-1\right)\right]}{|z|^{N+2 s}} d x d z \\
-\iint \frac{Q(x)(Q(x)-Q(x-z))\left(1-\cos \left\langle z, v_{0}\right\rangle\right)}{|z|^{N+2 s}} d x d z,
\end{aligned}
$$

multiplied by $C(N, s) / 2$, represents a nonlocal counterpart of the total momentum in the local case, precisely (compare $\mathscr{E}_{1}$ and $\mathscr{E}_{2}$ with the right-hand side of [17, formula 3.5$]$ )

$$
-\left\langle\dot{x}(t), \int p_{\text {local }}^{\varepsilon}(t, x)\right\rangle, \quad p_{\text {local }}^{\varepsilon}(t, x):=\frac{1}{\varepsilon^{N-1}} \mathfrak{I m}\left(\bar{u}^{\varepsilon}(t, x) \nabla u^{\varepsilon}(t, x)\right), \quad x \in \mathbb{R}^{N}, t \in[0, \infty) .
$$

As known, $p_{\text {local }}^{\varepsilon}$ satisfies the following identities, for $t \geq 0$ and $x \in \mathbb{R}^{N}$,

$$
\frac{\partial}{\partial t} \frac{\left|u^{\varepsilon}(t, x)\right|^{2}}{\varepsilon^{3}}=-\operatorname{div}\left(p_{\text {local }}^{\varepsilon}(t, x)\right), \quad \frac{\partial}{\partial t} \int p_{\text {local }}^{\varepsilon}(t, x) d x=-\frac{1}{\varepsilon^{N}} \int \nabla V(x)\left|u^{\varepsilon}(t, x)\right|^{2} d x .
$$

In the fractional case, a counterpart of these identities seems hard to obtain.

4.1. Proof of Theorem 1.2. By Corollary 4.6 and by the characterization of the ground states as minima on the sphere of $L^{2}$, we have $0 \leq \mathcal{E}\left(\Psi^{\varepsilon}(t)\right)-\mathcal{E}(Q)=\mathscr{E}(t, \varepsilon, s)+\mathcal{O}\left(\varepsilon^{2}\right)$, where $\mathscr{E}$ satisfies $\mathscr{E}(0, \varepsilon, s)=\mathcal{O}\left(\varepsilon^{2}\right)$. By Theorem 1.1 we know that there exist constants $B, C>0$ such that for $\phi \in H^{1}\left(\mathbb{R}^{3}, \mathbb{C}\right)$ with $\|\phi\|_{2}=\|Q\|_{2}$, we have

$$
\mathcal{E}(\phi)-\mathcal{E}(Q) \geq C \inf _{x \in \mathbb{R}^{3}, \theta \in[0,2 \pi)}\left\|\phi-e^{i \theta} Q(\cdot-x)\right\|_{H^{s}}^{2}
$$

provided that $\mathcal{E}(\phi)-\mathcal{E}(Q) \leq B$. Then, introducing

$$
T^{\varepsilon, s}:=\sup \left\{t \in\left[0, T_{0}\right] \mid \mathscr{E}(\tau, \varepsilon, s) \leq B \text { for all } \tau \in[0, t]\right\}
$$

and, since $\mathscr{E}(0, \varepsilon, s)=\mathcal{O}\left(\varepsilon^{2}\right)$, it follows that $T^{\varepsilon, s}>0$ for any $\varepsilon>0$ sufficiently small and every $s \in(0,1)$ there exist families of continuous functions $\theta^{\varepsilon, s}: \mathbb{R} \rightarrow[0,2 \pi)$ and $z^{\varepsilon, s}: \mathbb{R}^{N} \rightarrow \mathbb{R}$ which satisfy the assertion.

4.2. Proof of Theorem 1.3. For $s \in(0,1]$, consider the solution $u_{s}^{\varepsilon}(t, \cdot) \in H^{s}\left(\mathbb{R}^{N}, \mathbb{C}\right)$ to the Cauchy problem (1.1) Then, taking [7, Proposition 2.2 and Lemma 5.3] into account, there exists a positive constant $C$ such that

$$
\left\|u_{s}^{\varepsilon}(t)-Q_{s}\left(\frac{x-x_{s}(t)}{\varepsilon}\right) e^{\mathrm{i} \frac{\left\langle v_{s}(t), x\right\rangle}{\varepsilon}}\right\|_{\mathcal{H}_{\varepsilon}^{s}}^{2} \leq C \sum_{i=1}^{4} \mathbb{A}_{i}(t ; \varepsilon, s),
$$


where we have set

$$
\begin{aligned}
& \mathbb{A}_{1}(t ; \varepsilon, s):=\left\|u_{s}^{\varepsilon}(t)-u_{1}^{\varepsilon}(t)\right\|_{\mathcal{H}_{\varepsilon}^{s}}^{2}, \\
& \mathbb{A}_{2}(t ; \varepsilon, s):=\frac{1}{\varepsilon^{2(1-s)}}\left\|u_{1}^{\varepsilon}(t)-Q_{1}\left(\frac{x-x_{1}(t)}{\varepsilon}\right) e^{\mathrm{i} \frac{\left\langle v_{1}(t), x\right\rangle}{\varepsilon}}\right\|_{\mathcal{H}_{\varepsilon}^{1}}^{2}, \\
& \mathbb{A}_{3}(t ; \varepsilon, s):=\left\|Q_{1}\left(\frac{x-x_{s}(t)}{\varepsilon}\right) e^{\mathrm{i} \frac{\left\langle v_{s}(t), x\right\rangle}{\varepsilon}}-Q_{1}\left(\frac{x-x_{1}(t)}{\varepsilon}\right) e^{\mathrm{i} \frac{\left\langle v_{1}(t), x\right\rangle}{\varepsilon}}\right\|_{\mathcal{H}_{\varepsilon}^{s}}^{2}, \\
& \mathbb{A}_{4}(t ; \varepsilon, s):=\left\|Q_{s}\left(\frac{x-x_{s}(t)}{\varepsilon}\right)-Q_{1}\left(\frac{x-x_{s}(t)}{\varepsilon}\right)\right\|_{\mathcal{H}_{\varepsilon}^{s}}^{2},
\end{aligned}
$$

over finite time intervals $[0, T]$, for $T>0$. Then, we have the following

Proposition 4.8. There results

(a) $\mathbb{A}_{2}(t ; \varepsilon, s) \leq C \varepsilon^{2 s}$ for every $\varepsilon \in(0,1], s \in(0,1), t \geq 0$ and some $C>0$;

(b) $\lim _{s \rightarrow 1^{-}} \mathbb{A}_{3}(t ; \varepsilon, s)=0$ for every $\varepsilon \in(0,1]$ and $t \geq 0$;

(c) $\lim _{s \rightarrow 1^{-}} \mathbb{A}_{4}(t ; \varepsilon, s)=0$ for every $\varepsilon \in(0,1]$ and $t \geq 0$.

Proof. The proof of (a) follows immediately from [17, Theorem 1.1]. The proof of (b) is a consequence of the fact that $x_{s}(t) \rightarrow x_{1}(t)$ and $v_{s}(t) \rightarrow v_{1}(t)$ when $s \rightarrow 1$, since

$$
\begin{aligned}
\mathbb{A}_{3}(t, \varepsilon, s) & \leq C\left\|Q_{1}\left(\frac{\cdot-x_{s}(t)}{\varepsilon}\right)-Q_{1}\left(\frac{\cdot-x_{1}(t)}{\varepsilon}\right)\right\|_{\mathcal{H}_{\varepsilon}^{s}}^{2}+\left\|Q_{1}\left(\frac{\cdot-x_{1}(t)}{\varepsilon}\right)\left[e^{\mathrm{i} \frac{\left\langle v_{s}(t), x\right\rangle}{\varepsilon}}-e^{\mathrm{i} \frac{\left\langle v_{1}(t), x\right\rangle}{\varepsilon}}\right]\right\|_{\mathcal{H}_{\varepsilon}^{s}}^{2} \\
& =C\left\|Q_{1}(\cdot)-Q_{1}\left(\cdot+\frac{x_{s}(t)-x_{1}(t)}{\varepsilon}\right)\right\|_{H^{s}}^{2}+\left\|Q_{1}(\cdot) \Xi_{s}(\cdot, t)\right\|_{H^{s}}^{2} \\
& \leq C\left\|Q_{1}(\cdot)-Q_{1}\left(\cdot+\frac{x_{s}(t)-x_{1}(t)}{\varepsilon}\right)\right\|_{H^{1}}^{2}+C\left\|Q_{1}(\cdot) \Xi_{s}(\cdot, t)\right\|_{H^{1}}^{2},
\end{aligned}
$$

where we have set

$$
\Xi_{s}(x, t):=e^{\mathrm{i}\left\langle v_{s}(t), x+\varepsilon^{-1} x_{1}(t)\right\rangle}-e^{\mathrm{i}\left\langle v_{1}(t),\left(x+\varepsilon^{-1} x_{1}(t)\right\rangle\right.}, \quad t \geq 0, x \in \mathbb{R}^{N} .
$$

The first term goes to zero as $s \rightarrow 1^{-}$, for any $\varepsilon \in(0,1]$ and $t \geq 0$ (see e.g. [17, p.185]). Since $\left|\Xi_{s}(x, t)\right| \leq 2$ and $\left|\nabla \Xi_{s}(x, t)\right| \leq\left\|v_{s}\right\|_{L^{\infty}(0, T)}+\left\|v_{1}\right\|_{L^{\infty}(0, T)}$, the second term goes to zero by dominated convergence. The proof of (c) is a direct application of [9, Lemma 2.6], since

$$
\mathbb{A}_{4}(t, \varepsilon, s)=\left\|Q_{s}\left(\frac{x-x_{s}(t)}{\varepsilon}\right)-Q_{1}\left(\frac{x-x_{s}(t)}{\varepsilon}\right)\right\|_{\mathcal{H}_{\varepsilon}^{s}}^{2}=\left\|Q_{s}-Q_{1}\right\|_{H^{s}}^{2},
$$

concluding the proof.

Based upon the previous conclusions, the proof of Theorem 1.3 is complete.

\section{REFERENCES}

[1] V. Benci, M.G. Ghimenti, A.M. Micheletti, The nonlinear Schrödinger equation: soliton dynamics, J. Differential Equations 249 (2010), 3312-3341. 1

[2] V. Benci, M.G. Ghimenti, A.M. Micheletti, On the dynamics of solitons in the nonlinear Schrödinger equation, Arch. Ration. Mech. Anal. 205 (2012), 467-492. 1

[3] J.C. Bronski, R.L. Jerrard, Soliton dynamics in a potential, Math. Res. Lett. 7 (2-3) (2000), 329-342. 1

[4] X. CABre, Y. Sire, Nonlinear equations for fractional Laplacians II: existence, uniqueness, and qualitative properties of solutions, Trans. Amer. Math. Soc., to appear. 2

[5] T. Cazenave, P.L. Lions, Orbital stability of standing waves for some nonlinear SchrÃüdinger equations, Comm. Math. Phys. 85 (1982), 549-561. 16

[6] P. D'Avenia, M. Squassina, Soliton dynamics for the Schrödinger-Newton system, Math. Models Methods Appl. Sci., to appear. 2

[7] E. Di Nezza, G. Palatucci, E. Valdinoci, Hitchhiker's guide to the fractional Sobolev spaces, Bull. Sci. math. 136 (2012), 512-573. 5, 17, 18, 20

[8] D.E. Edmunds, W.D. Evans, Spectral theory and differential operators, Clarendon Press, 1987. 15

[9] M.M. Fall, E. Valdinoci, Uniqueness and nondegeneracy of positive solutions of $\Delta u+u=u^{p}$ in $\mathbb{R}^{N}$ when $s$ is close to 1 , preprint. $6,16,21$ 
[10] P. Felmer, A. Quahs, J. Tan, Positive solutions of nonlinear Schrödinger equation with the fractional Laplacian, Proc. Royal Soc. Edinburgh A 142 (2012), 1237-1262. 2

[11] R.L. Frank, E. Lenzmann, Uniqueness and non degeneracy of ground states for $(-\Delta)^{s} Q+Q-Q^{\alpha+1}=0$ in $\mathbb{R}$. Acta Math., to appear. 2, 11, 13

[12] R.L. Frank, E. Lenzmann, L. Silvestre, Uniqueness of radial solutions for the fractional laplacian, preprint. http://arxiv.org/abs/1302.2652. 2, 6, 13

[13] J. Fröhlich, S. Gustafson, B.L.G. Jonsson, I.M. Sigal, Dynamics of solitary waves external potentials, Comm. Math. Phys. 250 (2004), 613-642. 1

[14] B. Guo, D. Huang, Existence and stability of standing waves for nonlinear fractional Schrödinger equations, J. Math. Phys 53083702 (2012). 2

[15] B. Guo, Z. Huo, Global well-posedness for the fractional nonlinear Schrodinger equation, Comm. Partial Differential Equations 36 (2011), 247-255. 2

[16] R. Hilfer, Application of fractional calculus in physics, World Scientific, Singapore, 2000. 1

[17] S. Keraani, Semiclassical limit for nonlinear Schrödinger equation with potential II, Asymptot. Anal. 47 (2006), 171-186. 1, 20, 21

[18] R. Killip, M. VisAn, Nonlinear Schrödinger equations at critical regularity, lectures delivered at Clay Institute, 2008. Available at http://www.claymath.org/programs/summer_school/2008/visan.pdf 3

[19] N. Laskin, Fractional Schrödinger equations, Phys. Rev. E 66 (2002), 056108. 1

[20] E. Lenzmann, Uniqueness of ground states for pseudorelativistic Hartree equations, Anal. PDE 2 (2009), 1-27. 2

[21] R. Metzler, J. Klafter, The random walkâĂŹs guide to anomalous diffusion: a fractional dynamics approach, Phys. Rep. 339 (2000), 1-77. 1

[22] R. Metzler, J. Klafter, The restaurant at the random walk: recent developments in the description of anomalous transport by fractional dynamics, J. Phys. A 37 (2004), 161-208. 1

[23] X. Ros-Oton, J. Serra, The Pohozaev identity for the fractional laplacian, preprint http://arxiv.org/abs/1207.5986 11

[24] P. Rozmej, B. Bandrowski, On fractional Schrödinger equation, Comput. Methods Sci. Technol. 16 (2010), 191-194. 1

[25] T. TAO, Why are solitons stable? Bull. Amer. Math. Soc. 46 (2009), 1-33. 2

[26] G. Teschl, Mathematical methods in Quantum Mechanics, with applications to Schrödinger operators. Graduate Studies in Mathematics 99, American Mathematical Society, 2009. 13

[27] J. Weidmann, Linear operators in Hilbert spaces. Graduate Texts in Mathematics 68. Springer, 1980. 10, 13

[28] M.I. Weinstein, Modulational stability of ground states of nonlinear Schrödinger equations, SIAM J. Math. Anal. 16 (1985) 472-491. 1, 2

[29] M.I. Weinstein, Lyapunov stability of ground states of nonlinear dispersive evolution equations, Comm. Pure Appl. Math. 39 (1986), 51-67. 1, 2

Dipartimento di Matematica e Applicazioni

Universitì di Milano BicoCCA

Edificio U5, Via Roberto Cozzi 53, 20125 Milano, Italy

E-mail address: simone.secchi@unimib.it

Dipartimento Di InFormatica

UNIVERSitÀ DEGLi StUdi di VeronA

Cá Vignal 2, Strada Le Grazie 15, 37134 Verona, Italy

E-mail address: marco.squassina@univr.it 